\title{
Coherent manipulation of non-thermal spin order in optical nuclear polarization experiments
}

Gerd Buntkowsky, Konstantin L. Ivanov, Herbert Zimmermann, and Hans-Martin Vieth

Citation: The Journal of Chemical Physics 146, 114501 (2017); doi: 10.1063/1.4976990

View online: http://dx.doi.org/10.1063/1.4976990

View Table of Contents: http://aip.scitation.org/toc/jcp/146/11

Published by the American Institute of Physics

\section{Articles you may be interested in}

Effect of the reflectional symmetry on the coherent hole transport across DNA hairpins

The Journal of Chemical Physics 146, 114105114105 (2017); 10.1063/1.4978571

Announcement: Top reviewers for The Journal of Chemical Physics 2016

The Journal of Chemical Physics 146, 100201100201 (2017); 10.1063/1.4978399

Grand canonical electronic density-functional theory: Algorithms and applications to electrochemistry

The Journal of Chemical Physics 146, 114104114104 (2017); 10.1063/1.4978411

Intermolecular potential energy surface and thermophysical properties of propane The Journal of Chemical Physics 146, 114304114304 (2017); 10.1063/1.4978412

From the tunneling dimer to the onset of microsolvation: Infrared spectroscopy of allyl radical water aggregates in helium nanodroplets

The Journal of Chemical Physics 146, 114306114306 (2017); 10.1063/1.4978482

On the non-exponentiality of the dielectric Debye-like relaxation of monoalcohols

The Journal of Chemical Physics 146, 114502114502 (2017); 10.1063/1.4978585

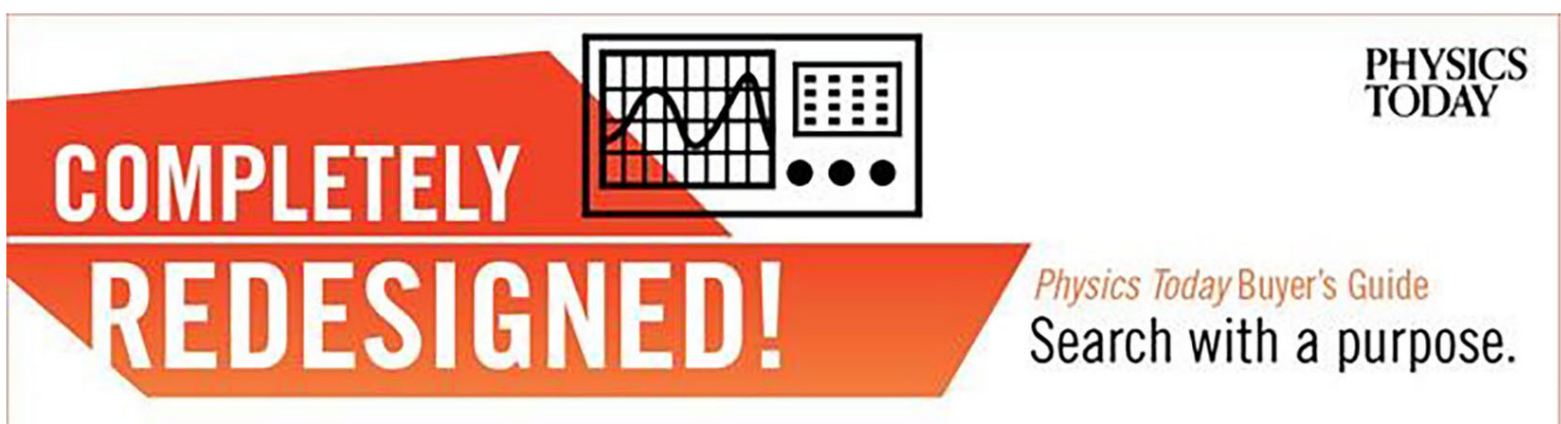




\title{
Coherent manipulation of non-thermal spin order in optical nuclear polarization experiments
}

\author{
Gerd Buntkowsky, ${ }^{1}$ Konstantin L. Ivanov, ${ }^{2,3}$ Herbert Zimmermann, ${ }^{4}$ \\ and Hans-Martin Vieth ${ }^{2,5}$ \\ ${ }^{1}$ Fachbereich Chemie, Technische Universität Darmstadt, Alarich-Weiss-Str. 8, Darmstadt 64287, Germany \\ ${ }^{2}$ International Tomography Center, Siberian Branch of the Russian Academy of Science, Institutskaya $3 a$, \\ Novosibirsk 630090, Russia \\ ${ }^{3}$ Novosibirsk State University, Pirogova 2, Novosibirsk 630090, Russia \\ ${ }^{4}$ Max-Planck-Institut für Medizinische Forschung, Heidelberg, Jahnstr. 29, 69120 Heidelberg, Germany \\ ${ }^{5}$ Institut für Experimentalphysik, Freie Universität Berlin, Arnimallee 14, 14195 Berlin, Germany
}

(Received 21 November 2016; accepted 7 February 2017; published online 17 March 2017)

\begin{abstract}
Time resolved measurements of Optical Nuclear Polarization (ONP) have been performed on hyperpolarized triplet states in molecular crystals created by light excitation. Transfer of the initial electron polarization to nuclear spins has been studied in the presence of radiofrequency excitation; the experiments have been performed with different pulse sequences using different doped molecular systems. The experimental results clearly demonstrate the dominant role of coherent mechanisms of spin order transfer, which manifest themselves in well pronounced oscillations. These oscillations are of two types, precessions and nutations, having characteristic frequencies, which are the same for the different molecular systems and the pulse sequences applied. Hence, precessions and nutations constitute a general feature of polarization transfer in ONP experiments. In general, coherent manipulation of spin order transfer creates a powerful resource for improving the performance of the ONP method, which paves the way to strong signal enhancement in nuclear magnetic resonance. Published by AIP Publishing. [http://dx.doi.org/10.1063/1.4976990]
\end{abstract}

\section{INTRODUCTION}

Nuclear Magnetic Resonance (NMR) is one of the most attractive spectroscopic techniques, which is applied in various fields of research ranging from physics, material science, and chemistry to biology and medicine. Despite their exquisite spectral sensitivity to structure, dynamics, and morphology, conventional NMR methods suffer from a notoriously low detection sensitivity limiting their power and applicability. Unlike in optical and other spectroscopic methods, wherein the entire ensemble (or large fractions of the ensemble) give rise to signal formation, conventional NMR represents a case where the energy level spacing is much lower than the ambient thermal energy $(k T)$, and thus the involved energy levels are nearly equally populated. Consequently, the polarization of spins at thermal equilibrium is low (for instance, only $3 \times 10^{-5}$ for protons at room temperature and a magnetic field of $10 \mathrm{~T}$ ): since the NMR signal is directly proportional to polarization, this leads to an enormous loss in the signal intensity. ${ }^{1}$ On the other hand, when spins are shifted from thermal equilibrium, i.e., when they are hyper-polarized, huge NMR signal enhancements, up to several orders of magnitude, are expected. Accordingly, there have been several hyperpolarization techniques established. The oldest and particularly popular technique is Dynamic Nuclear Polarization (DNP) ${ }^{2-5}$ which is based on the transfer of the much higher thermal polarization of electron spins to the nuclear spin ensemble by pumping Electron Paramagnetic Resonance (EPR) transitions of paramagnetic dopants (or conduction electrons) in the sample. DNP enables signal enhancements given by the ratio of the electron and nuclear gyromagnetic ratios, i.e., reaching approximately 660 for protons and even more for other popular NMR nuclei, e.g., ${ }^{13} \mathrm{C}$ and ${ }^{15} \mathrm{~N}$. A closely related hyperpolarization method is Optical Nuclear Polarization (ONP), ${ }^{6-8}$ which exploits hyperpolarized triplet states formed by light excitation and subsequent polarization transfer to surrounding nuclei in a suitable matrix during the electron-nuclear "spin contact." Such triplet states can be formed in molecular crystals doped with guest molecules by electron-spin selective Inter-System Crossing (ISC). ONP can also be observed in other systems, e.g., in semiconductors ${ }^{9,10}$ and defect centers in diamond crystals. ${ }^{11,12}$ One should note that the ONP method is sometimes termed "triplet-state DNP" 13,14 or "triplet-state CIDNP"; 15 however, here we would like to use the term "ONP" to discriminate clearly between related, but different hyperpolarization methods. Importantly, in ONP the initial electron spin polarization can be close to $100 \%$, i.e., stronger than the thermal electron spin polarization in DNP experiments (unless DNP experiments are performed at very low temperature $\left.{ }^{16}\right)$. Moreover, experiments can be run at room temperature; ${ }^{17}$ this makes ONP an attractive method for creating hyper-polarized spins. However, for reaching high performance of ONP and achieving strong NMR signal enhancement, the polarization transfer step must be optimized, i.e., the starting electron spin order has to be transferred to the target nuclei as efficiently as possible.

Polarization transfer is a frequent task in magnetic resonance, emerging both in NMR and EPR in various aspects, and in hyperpolarization. Polarization transfer is based either on stochastic (cross-relaxation; prominent examples are the 
Overhauser-type $\mathrm{DNP}^{18}$ and the nuclear Overhauser effect ${ }^{19}$ ) or on coherent mechanisms. In general, coherent mechanisms are known to be faster and by far more efficient; therefore, in ONP it is highly advantageous to exploit coherent polarization transfer from the hyper-polarized triplet states to the nuclear spins. In other hyperpolarization methods the power of coherent transfer mechanisms has been demonstrated as well, notably in solid-effect ${ }^{20,21}$ and cross-effect DNP. ${ }^{22,23}$ To have a better "spin contact" in ONP and to exploit spin coherences, specific matching conditions are required, which can be fulfilled either at crossings ${ }^{24}$ of electron-nuclear spin energy levels or upon excitation of the EPR transitions ${ }^{25-27}$ of the triplet state. In this work, the second option is pursued for optimizing polarization transfer.

Hence, here we demonstrate coherent phenomena in ONP; coherent behavior clearly manifests itself in an oscillatory time dependence of the spin hyperpolarization process. The presence of such oscillations also has influence on interpretation of the ONP mechanism: in contrast to earlier works suggesting a thermodynamic description of ONP in terms of spin reservoirs exchanging energy, ${ }^{28}$ we show that only a microscopic quantum-mechanical description is suitable for the description of the observed polarization transfer phenomena. Here, we limit ourselves only to a semi-qualitative interpretation of the observed coherent effects in ONP experiments; the quantitative description will be presented elsewhere. In this work, we present additional experimental data for time-resolved studies on suitable model systems, thereby showing that previous observations of coherent oscillations of ONP obtained by some of us ${ }^{29}$ hold for a variety of experimental systems. Furthermore, we demonstrate that in all systems studied here such oscillations can be classified as precessions and nutations: this general behavior is thus a characteristic for ONP.

It is worth noting that ONP is not only a method of hyperpolarizing nuclear spins but it also gives a way to study the general nature of spin order transfer phenomena in systems of coupled electronic and nuclear spins. Since these transfer processes are dynamic, coherent processes, their investigation requires time-resolved experiments. Due to the presence of electronic spins with high magnetic moments, such an experiment requires time resolution on the nanosecond time scale, imposing tough experimental demands. Nonetheless, the ONP technique is entirely suitable for running such time-resolved experiments, as shown here. In addition, ONP is dealing with non-thermal electronic polarization and can provide very high NMR enhancement at relatively high temperature and relatively low field, when in DNP the enhancements are not the highest. Furthermore, ONP is operative in single crystals so that polarization transfer experiments can be run using specific and well-defined orientation of the spin interaction tensors to study the spin evolution in detail. While we deal here exclusively with polarization of protons, ONP can straightforwardly be extended to other nuclei such as ${ }^{2} \mathrm{H}$ and ${ }^{13} \mathrm{C}$.

\section{METHODS}

\section{A. Experimental method}

A class of systems, which are suitable for our ONP studies, is given by molecular crystals doped with optically active guest molecules in low concentration. Instead of employing thermal electron spin polarization, the guest molecules are excited by light irradiation from their $\mathrm{S}_{0}$ ground state to a higher singlet state. From here, a part of the population proceeds by intersystem crossing (ISC) to the lowest triplet state, whose sub-levels are selectively populated. ${ }^{7,8}$ In contrast to the Boltzmann polarization, this Optical Electron Polarization (OEP) is independent of the Zeeman energy of the triplet spins and of the temperature, which allows one to generate electron spin polarization of about $90 \%$ even at room temperature in low magnetic fields. The triplet concentration can be varied over several orders of magnitude via the dopant concentration as well as by the light intensity. Since the optical excitation process occurs on a time scale, which is much shorter than the time scale of $10 \mathrm{~ns}$ relevant for spin-spin couplings, pulsed light irradiation enables forming of the electronic spin polarization nearly instantaneously for all individual triplet sub-states.

The transfer of this OEP to the nuclear spins in the matrix is commonly termed ONP and constitutes the experimental method used in our investigations. The first timeresolved experiments of this kind were performed in Refs. 30-32 and enabled direct observation of coherent processes in the electron-nuclear polarization transfer, which reveal themselves in the oscillatory time dependence of the resulting nuclear spin polarization. In contrast to the experiments at high external polarization fields of about $300 \mathrm{mT},{ }^{31}$ which show no pronounced oscillations, it turned out that our measurements in low external fields of about $30 \mathrm{mT}$, which are comparable to the local hyperfine fields, show clearly pronounced oscillations. ${ }^{30}$ Since these oscillations appear to play the key role in the polarization transfer mechanism, we have extended our low field experiments by systematic variation of external parameters, the pulse sequences, and the molecular systems.

The key steps of the ONP experiment are depicted in Figure 1. The experiment starts by optical excitation of an appropriate guest molecule from its electronic ground state $S_{0}$ into a higher singlet state $S_{1}$. These excited molecules have different decay channels, one of which leads via ISC to the lowest triplet state $T_{1}$ of the molecule. Due to symmetry selection rules, the ISC leads to preferential population of a single spin sub-level of the triplet manifold. This corresponds to a high electronic spin order of the triplet state, but has no influence on the nuclear polarization. By irradiating the triplet spin system with high-frequency fields near the resonance of two of the three triplet sub-levels, transfer of the electron spin polarization to neighboring nuclear spins in the matrix becomes possible. This transfer pathway is symbolized by the "switch" in Figure 1. The subsequent decay

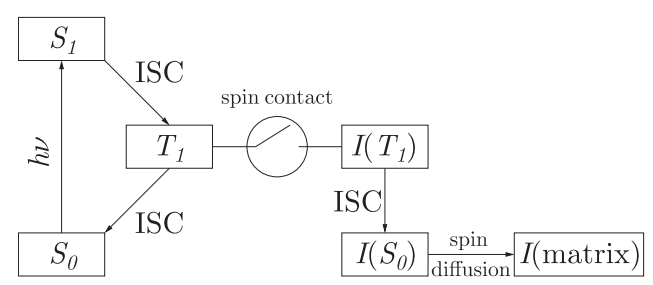

FIG. 1. General scheme of the optical nuclear polarization (ONP) mechanism. See text for discussion. 
of the triplet states has no influence on the nuclear polarization. Due to the low concentration and short lifetime of the triplet states, the nuclear spin polarization achieved is concentrated initially in the proximity of the individual triplet states. To enhance the total nuclear polarization, this ONP cycle is repeated several times. Between the individual irradiation cycles, spin diffusion ${ }^{33-35}$ within the nuclear spin system is operative, distributing the locally created nuclear spin polarization uniformly over the whole sample. In contrast to the DNP case, ${ }^{36,37}$ in ONP we hardly have any "spin diffusion barrier" because the ground state of the spin system is diamagnetic. Consequently, the nuclei experience no shifts of their NMR lines and freely exchange polarization. As all paramagnetic species decay fast, nuclear spin-lattice relaxation is slow; hence, the polarization process can be repeated until a sufficiently high ONP is achieved, which enables strong NMR signal enhancement and, consequently, direct NMR detection within a single acquisition.

\section{B. Experimental arrangements and procedure}

In this section, we give a brief description of the apparatus used to investigate the ONP mechanism, as depicted in Figure 1.

A typical ONP measurement consists of three consecutive stages:

\section{Preparation stage}

The experiment starts by destroying residual spin order of the nuclear spin system by a train of $\pi / 2$ NMR pulses at the detection field $B_{\text {det }}$, here $B_{\text {det }}=705 \mathrm{mT}$ ( $30 \mathrm{MHz}$ proton NMR frequency). After this the field is switched to the polarization field $B_{p o l}$ typically in the range $10-100 \mathrm{mT}$. This is done by varying the current in an electromagnet employing a control loop with a Hall sensor.

\section{Polarization stage}

This stage comprises a cycle of three steps:

(a) Generation of the electronic triplet spin order by pulsed light irradiation.

(b) Application of a radio-frequency ( $\mathrm{RF}$ ) pulse sequence, see below, close to a single quantum transition of the triplet spin system.

(c) The initial triplet spin polarization is transferred only to nuclear spins in the vicinity of the individual triplet spins. After the end of the RF-irradiation and triplet decay, this local degree of spin order is distributed over the whole nuclear spin system by spin diffusion, on the time scale of several $10 \mathrm{~ms}$. This mechanism allows repetition of the polarization step and accumulation of the created nuclear spin order over several irradiation cycles.

Hence, the cycle 2(a)-2(c) is repeated with a repetition rate of $10-50 \mathrm{~Hz}$ for $1-100 \mathrm{~s}$ under identical conditions, until a sufficiently high nuclear spin polarization is obtained.

\section{Detection stage}

At the end of the polarization phase, the external magnetic field is adiabatically increased during approximately $10 \mathrm{~s}$ from the polarization field $B_{p o l}$ to the detection field $B_{d e t}$. Finally, the achieved nuclear polarization is detected by measuring the initial amplitude of the free induction decay by NMR.

The acquisition of a single ONP spectrum (corresponding to specific conditions for electron-nuclear polarization transfer) requires typically about $1 \mathrm{~min}$, while a complete ONP measurement consists typically of about 100 of these discreet points. Further detail of the experimental procedure is given in the supplementary material, specifically, the block diagram of the experimental setup and typical parameter settings.

\section{Molecular systems and sample preparation}

In order to investigate to what extent the experimental results depend on specific properties of the systems under investigation, we performed our experiments using two different molecular systems: pentacene guest molecules doped into a naphthalene matrix $(\mathrm{Pe} / \mathrm{Na})$ and acridine guest molecules doped into a fluorene matrix (Ac/Fl). The choice of these systems was motivated by the availability of all relevant system parameters from literature. Details of the crystal structure are given in Refs. 38 and 39 for $\mathrm{Pe} / \mathrm{Na}$ and are summarized in Ref. 8 for $\mathrm{Ac} / \mathrm{Fl}$.

The crystals were grown from the melt by the Bridgman technique. The dopant concentration in the melt for $\mathrm{Pe} / \mathrm{Na}$ and $\mathrm{Ac} / \mathrm{Fl}$ was $100 \mathrm{ppm}$ and $2000 \mathrm{ppm}$, respectively. The crystals contain a reduced guest concentration, which is for pentacene in naphthalene about $10 \mathrm{ppm} .{ }^{40}$ We denote the long in-plane axis of the guest molecules by $\hat{x}_{m}$, the short in-plane axis by $\hat{y}_{m}$, and the axis perpendicular to the plane by $\hat{z}_{m}$. Both systems feature two different sites for the guest molecules. Since these sites become magnetically equivalent for the orientation where the $\hat{x}_{m}$-axis is parallel to the external magnetic field, we have chosen this particular orientation in our experiments. Both systems exhibit the $a b$-plane as the unique well pronounced cleavage plane. Using cross-polarized light, the principal axis system of the index ellipsoid can be directly determined. These axes coincide with the crystalline $\hat{a}$ and $\hat{b}$ axis, respectively, in the $a b$-plane. The ratio of the absorption of light polarized along the $\hat{a}$ and $\hat{b}$-axis is $2: 13$ for $\mathrm{Pe} / \mathrm{Na},{ }^{41}$ which can be used to distinguish between these two axes. Since $\hat{x}_{m}$ is perpendicular to the crystalline $\hat{b}$-axis for $\mathrm{Pe} / \mathrm{Na}$, the crystal $\hat{b}$-axis is chosen as the rotation axis in the experiment. In the system Ac/Fl, $\hat{x}_{m}$ lies perpendicular to the $a b$-plane and is parallel to the crystal $\hat{c}$-axis. This allows us to choose any axis in the $a b$-plane to be taken as the axis of rotation.

\section{RESULTS}

In this section, we present a comprehensive survey of our experimental results. Subsections III A and III B are devoted to the characterization of the systems. The dependencies of the signals on the external fields $B_{p o l}$ and $B_{1}$ are of relevance for the polarization transfer efficiency. Lifetime measurements of the electron spin polarization allow one to determine the time interval, in which the polarization transfer can be observed, and indicate also to what extent relaxation is of importance. After such a characterization, we present the results of timeresolved experiments of the polarization transfer using a variety of different pulse sequences and experimental conditions. The discussion and interpretation of the experimental results 
require the knowledge of all experimental details and will be done in Sec. IV A.

\section{A. ONP field dependence}

RF-irradiation is applied resonant to two of the three triplet sub-levels. Due to structural inhomogeneities, different local fields, and relaxation processes, the resonance positions of the individual triplet states feature a distribution, which is henceforth called the EPR-line, $g(\omega)$. Several relevant characteristics of this distribution are seen in Figure 2 where the proton polarization resulting from irradiation by a single pulse of a fixed length is shown as a function of the external polarization field $B_{p o l}$. In this case, the pulse length was taken so long that oscillatory signal components are irrelevant: oscillatory components starting at different times, i.e., having different phases, are washed out. The pulse amplitude was reduced until no RF-related broadening was observed.

Polarization transfer under such experimental conditions results in the ONP field dependence given (roughly) by the derivative of $g(\omega)$ with respect to the external magnetic field. The zero crossing point corresponds to the situation where the center $\omega_{0}=\Omega\left(\omega_{e}\right)$ of the EPR-line equals the RF-frequency, $\omega_{0}=\omega_{r f}$ where $\omega_{e}=\gamma_{e} \cdot B_{p o l}$ and $\Omega$ describes the energy spacing of the two coupled triplet sub-levels due to zero field and Zeeman interaction. Hereafter $\gamma_{e}$ and $\gamma_{n}$ are the gyromagnetic ratios of the electron and proton, respectively. The extrema in the ONP field dependence correspond to the maximum slope of the derivative of the EPR-line. The solid line in Figure 2 represents a fit function, which is the derivative of a Gaussian shaped EPR-line $g(\Delta) \propto \exp \left(-\frac{1}{2} \cdot \Delta^{2} / \sigma_{E P R}^{2}\right)$ of width $\sigma_{E P R}$; here $\Delta$ is the off-set from the center of the line. From the experimental results linewidths of $5 \pm 1 \mathrm{MHz}$ and $16 \pm 1.2 \mathrm{MHz}$ are obtained for deuterated $\left(\mathrm{Pe}-\mathrm{d}_{14}\right)$ and protonated $\left(\mathrm{Pe}-\mathrm{h}_{14}\right)$ pentacene, respectively, in a protonated naphthalene $\left(\mathrm{Na}-\mathrm{h}_{8}\right)$ matrix. The linewidth for Ac- $\mathrm{d}_{9} / \mathrm{Fl}-\mathrm{d}_{8} \mathrm{~h}_{2}$ is $9 \pm 1 \mathrm{MHz}$.

The contributions of the dipolar and contact hyperfine interaction to the width of the EPR-line can be approximated by the second moment of the distribution of these fields, $\sigma_{S I}=\sqrt{\sum_{i} \eta_{i}^{2} \cdot A_{z z, i}}$, where $A_{z z, i}$ is the secular part of the hyperfine interaction between a fictitious two-level triplet spin $S=1 / 2$ (see explanation in Sec. IV) and the $i$ th nuclear spin $I_{i}$ in its surrounding, and $\eta_{i}^{2}$ is $1 / 4$ for $I=1 / 2$ and $2 / 3$ for $I=1$.

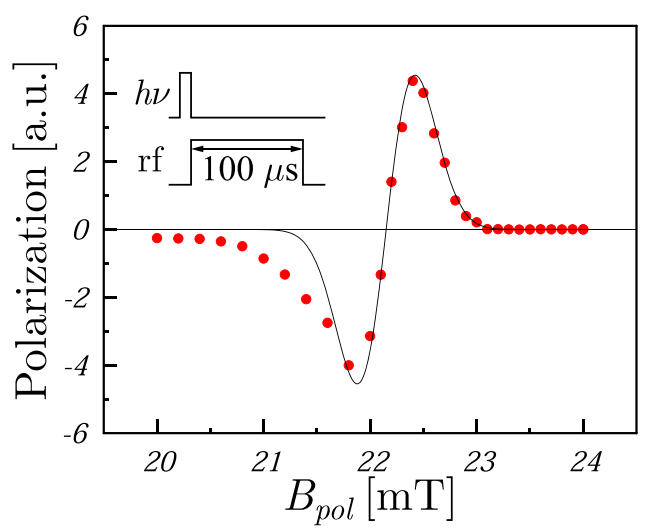

FIG. 2. Proton polarization as a function of the polarization field $B_{p o l}$. (System Pe-d ${ }_{14} / \mathrm{Na}-\mathrm{h}_{8}, \frac{\omega_{r f}}{2 \pi}=140 \mathrm{MHz}, \sqrt{2} \cdot \frac{\omega_{1}}{2 \pi}=1.2 \mathrm{MHz}, T=250 \mathrm{~K}$.)
The hyperfine coupling elements $A_{z z, i}$ are calculated using a simple dipole model as described in Ref. 42. Using the known crystal structure, the triplet spin densities, and the McConnell parameter of pentacene taken from Ref. 43, the hyperfine contributions are $\sigma_{S I}=15 \mathrm{MHz}$ and $4.0 \mathrm{MHz}$ for $\mathrm{Pe}-\mathrm{h}_{14} / \mathrm{Na}-\mathrm{h}_{8}$ and $\mathrm{Pe}-\mathrm{d}_{14} / \mathrm{Na}-\mathrm{h}_{8}$, respectively, where 200 neighboring naphthalene matrix molecules and one pentacene guest molecule were included in the calculations. Assuming a Gaussian distribution for the remaining contributions, $\sigma_{r}$, to $\sigma_{E P R}$ the experimental linewidths can be written as $\sigma_{E P R}=\sqrt{\sigma_{S I}^{2}+\sigma_{r}^{2}}$, where $\sigma_{r} \approx 3 \mathrm{MHz}$.

The signal shows a considerable deviation from the Gaussian shape on the low-field side. Direct EPR measurements show also a pure Gaussian shape of the EPR line ${ }^{44}$ with comparable linewidths. Since $\sigma_{E P R}$ is mainly determined by the local hyperfine fields, the relation between these fields and the external field will henceforth be given by the ratio of $\sigma_{E P R}$ and the external field in units of the nuclear Zeeman energy $\omega_{n}=\gamma_{n} \cdot B_{p o l}$. In this nomenclature $\sigma_{E P R}>\omega_{n}$ is called the low-field case and $\sigma_{E P R}<\omega_{n}$ is called the high-field case.

The dependence of the signal with respect to the amplitude $\omega_{1}=\gamma_{e} \cdot B_{1}$ of the RF-field is another characteristic, which can be directly compared with theoretical predictions. In Figure 3 two $\omega_{1}$ dependencies are shown for a single pulse, irradiated off-resonant at $\Delta \omega=\omega_{0}-\omega_{r f}=\sigma_{E P R}$. The pulse length $t_{p}$ was chosen in such a way that oscillatory signal components vanish. Independent of $t_{p}$, a maximum can be observed at $\sqrt{2} \cdot \omega_{1}=1.4 \pm 0.1 \mathrm{MHz}$ for $\omega_{n}=0.94 \mathrm{MHz}$. When going to $\omega_{n}=1.1 \mathrm{MHz}$ this maximum shifts to $\sqrt{2} \cdot \omega_{1}=1.7 \pm 0.1 \mathrm{MHz}$. For shorter pulses, a second, broader maximum is obtained at $\sqrt{2} \cdot \omega_{1} \approx 12 \mathrm{MHz}$. The position and amplitude of this maximum depend on $\omega_{n}$ and $t_{p}$. Unlike other measurements of this type in higher external magnetic fields $\left(\omega_{n}>10 \mathrm{MHz}\right)$, which show for comparatively long pulses ( $>5 \mu \mathrm{s}$ ) only a single maximum for $\sqrt{2} \cdot \omega_{1} \approx \omega_{n}, 31,45$ these measurements clearly indicate that for short pulses two maxima of different widths but of comparable strengths can be obtained.

Closer inspection of Figures 2 and 3 indicates that polarization transfer is most efficient when a matching condition is fulfilled, i.e., when the electron nutation frequency matches

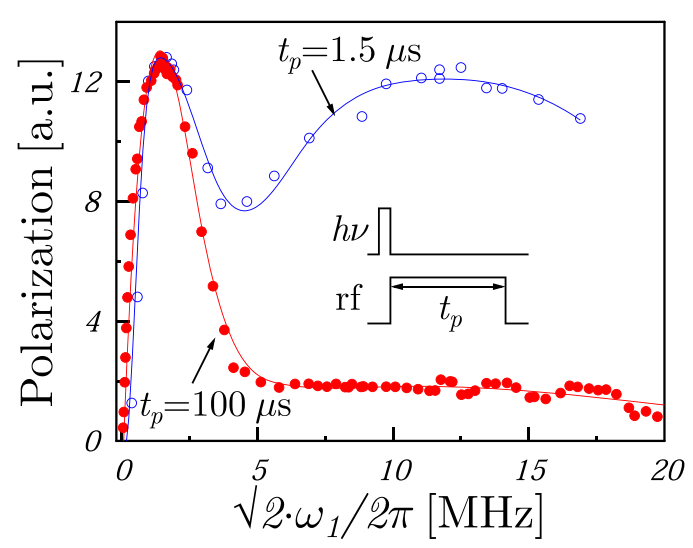

FIG. 3. Proton polarization as a function of the RF-amplitude $\omega_{1}=\gamma_{e} \cdot B_{1}$. The signals are normalized for the same maximal amplitude. (System Pe$\mathrm{d}_{14} / \mathrm{Na}-\mathrm{h}_{8}, \frac{\omega_{r f}}{2 \pi}=140 \mathrm{MHz}=\frac{\omega_{0}}{2 \pi}+\frac{\sigma_{E P R}}{2 \pi}, \frac{\omega_{n}}{2 \pi}=0.94 \mathrm{MHz}, T=250 \mathrm{~K}$.) Solid lines are drawn only to guide the eye. 
the nuclear precession frequency (see below). However, under these conditions the coherent nature of the polarization transfer is not clearly visible for the reasons explained later in the text. Therefore, hereafter we mainly show the data obtained when the spin system is away from the "matching condition." As we demonstrate below, in this situation the coherent spin dynamics has only two contributions, which behave qualitatively in the same way for both systems under investigation.

\section{B. Lifetime measurements}

For investigating the processes governing polarization transfer, it is of great importance to know to what extent decay and relaxation processes within the spin system influence the experimental results by affecting the lifetime of the OEP. This lifetime can be measured by delayed RF-irradiation with respect to the light excitation. Figure 4 shows two such measurements, where the applied pulse sequence is shown in the inset in Figure 4(a). Whereas the system Ac- $\mathrm{d}_{9} / \mathrm{Fl}-\mathrm{d}_{8} \mathrm{~h}_{2}$ shows an apparent mono-exponential decay of the electron polarization (Figure $4(\mathrm{~b})$ ), the system $\mathrm{Pe}-\mathrm{d}_{14} / \mathrm{Na}-\mathrm{h}_{8}$ shows an approximately bi-exponential decay (Figure 4(a)). The decay time constant for Ac- $\mathrm{d}_{9} / \mathrm{Fl}-\mathrm{d}_{8} \mathrm{~h}_{2}$ is $6.2 \pm 0.1 \mu \mathrm{s}$, which is in fair agreement with corresponding EPR measurements $\left(\omega_{r f}=9.4 \mathrm{GHz}\right)$ providing a decay time constant of $5.5 \pm 0.1 \mu \mathrm{s} .{ }^{46}$ For Pe- $\mathrm{d}_{14} / \mathrm{Na}-\mathrm{h}_{8}$, the two decay time constants are $\tau_{1}=35 \pm 5 \mu \mathrm{s}$ and $\tau_{2}=150 \pm 50 \mu \mathrm{s}$ as obtained from fitting. The large uncertainty of $\tau_{2}$ determination results from deviation from a pure bi-exponential behavior for $\tau>150 \mu \mathrm{s}$.

The amplitude of the proton polarization is proportional to the population difference between the two triplet sub-states coupled by the RF-field. The change of the sign of the signal
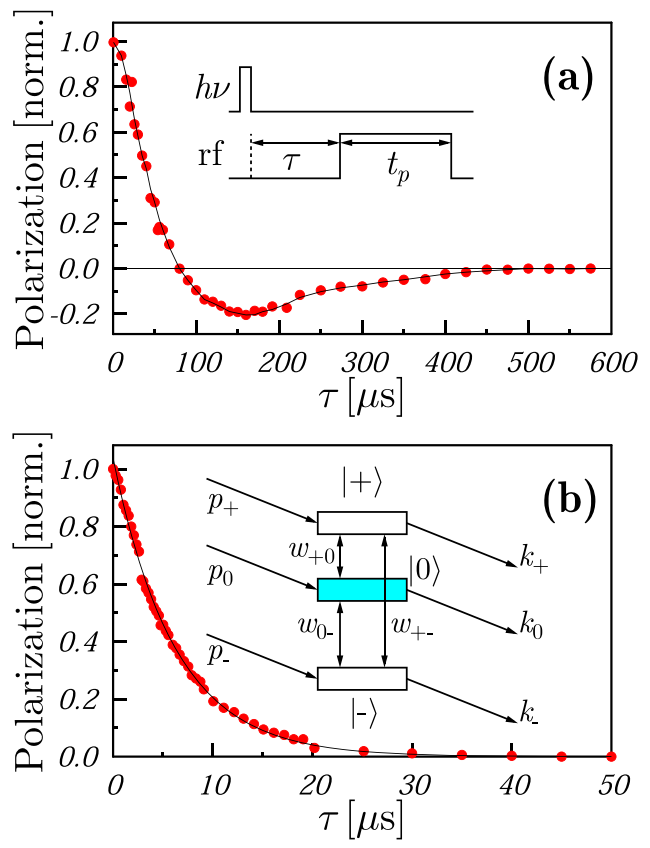

FIG. 4. Life time measurements of the electron spin polarization for (a) Pe-d ${ }_{14} / \mathrm{Na}-\mathrm{h}_{8}\left(\frac{\omega_{r f}}{2 \pi}=230 \mathrm{MHz}=\frac{\omega_{0}}{2 \pi}+\sigma_{E P R}, \sqrt{2} \cdot \frac{\omega_{1}}{2 \pi}=1.8 \mathrm{MHz}, T=250 \mathrm{~K}\right.$, $\left.t_{p}=100 \mu \mathrm{s}\right)$ and (b) Ac-d $/$ Fl-d $\mathrm{d}_{8} \mathrm{~h}_{2}\left(\frac{\omega_{r f}}{2 \pi}=207 \mathrm{MHz}=\frac{\omega_{0}}{2 \pi}+\frac{\sigma_{E P R}}{2 \pi}\right.$, $T=300 \mathrm{~K}$ ). The inset in (a) depicts the applied pulse sequence. The inset in (b) defines the notation for the energy levels and the rate constants within the triplet spin sub-levels. indicates therefore a population inversion in the triplet substates. The inversion point $\tau_{\text {inv }}$ for Pe- $\mathrm{d}_{14} / \mathrm{Na}-\mathrm{h}_{8}$ is about $80 \mu \mathrm{s}$. The values for $\tau_{1}, \tau_{2}$, and $\tau_{\text {inv }}$ are in agreement with corresponding EPR measurements (at $9.4 \mathrm{GHz}$ ) of Ong et al. ${ }^{47}$ on deuterated pentacene guest in a protonated $p$-terphenyl host. RF-irradiation is applied resonant to the field-independent sub-level $|0\rangle=\left|T_{x}\right\rangle$ and the field-dependent sub-level $|+\rangle$, where $|0\rangle$ and $| \pm\rangle$ are initially populated to $76 \%$ and $12 \%$, respectively. ${ }^{48}$ In this case $\tau_{1}$ and $\tau_{2}$ correspond to the decay constants: $k_{0}=\tau_{1}^{-1}, k_{+}=\tau_{2}^{-1}$. The appearance of the population inversion indicates hereby that the lifetime of the electron polarization is mainly determined by the triplet decay, where the relaxation $w_{i j}$ has only a minor influence, i.e., $w_{+0}<k_{+}, k_{0}$. The third sub-level has no observable influence on the polarization. The lifetime of the $|0\rangle$ sub-level of $35 \mu$ s for Pe- $\mathrm{d}_{14} / \mathrm{Na}-\mathrm{h}_{8}$ is about a factor of two larger than the lifetime of about $20 \mu \mathrm{s}$ for $\mathrm{Pe}-\mathrm{h}_{14} / \mathrm{Na}-\mathrm{h}_{8}{ }^{31}$ This variation due to deuteration is in agreement with the results of Ong et al. ${ }^{47}$ on pentacene in $p$-terphenyl.

The lifetime of the triplet state of Ac/Fl has been determined by observation of the Boltzmann signal in EPR experiments; it is in the order of a few ms. ${ }^{46}$ Hence, the lifetime of the triplet spin polarization is in this case determined solely by the spin-lattice relaxation rates within the triplet spin system.

From these measurements it becomes clear that the lifetime of the triplet spin polarization is of different origin in the two systems. Whereas the lifetime is determined for Pe/Na predominantly by the decay of the triplet states, the lifetime for $\mathrm{Ac} / \mathrm{Fl}$ is predominantly determined by relaxation processes. Moreover, the lifetimes differ by nearly an order of magnitude. Due to these facts, the comparison of these two systems allows a direct investigation of the influence of the two different types of polarization decay mechanisms on the polarization transfer.

Relaxation processes within the nuclear spin system determine via $T_{1 l}$ processes the time available for the accumulation of the achieved nuclear spin order during the polarization phase. $T_{1 l}$ denotes hereby the spin-lattice relaxation time of the protons under pulsed light irradiation. The proton polarization shows a mono-exponential behavior proportional to $\left\{\exp \left(-t / T_{1 l}\right)-1\right\}$, where $t$ is the total length of the irradiation and $T_{1 l}$ slightly depends on the repetition rate and the light intensity. Under typical experimental conditions with a repetition rate of $10-50 \mathrm{~Hz}, T_{1 l}$ is about $80 \mathrm{~s}$ for $\mathrm{Ac} / \mathrm{Fl}$. For Pe/Na $T_{1 l}$ is at room temperature at low fields (about $10 \mathrm{mT}$ ) less than $1 \mathrm{~s} .{ }^{49}$ By reducing the temperature slightly, the relaxation time $T_{1 l}$ increases dramatically and is of the order of several hours even at fields in the range of a few $10 \mathrm{mT}$ at temperatures below $250 \mathrm{~K} .{ }^{49}$ For this reason, experiments on this system were performed in the temperature range of 100-250 K where effects coming from $T_{1 l}$ can be neglected for typical irradiation times of about $100 \mathrm{~s}$.

\section{Single pulse experiments}

Without RF-irradiation, no ONP is obtained (see, e.g., Figure 3), unless the spin system is at a level crossing. By applying RF-irradiation, strong nuclear polarization can be obtained. By varying the length of the applied RF-pulses, it is possible to 
obtain information about the time evolution of the electronnuclear polarization transfer during RF-irradiation. Figure 5 shows three corresponding experiments under variation of the length of a single pulse for different RF-amplitudes $\omega_{1}$, with off-resonant irradiation, $\Delta \omega=\sigma_{E P R}$. In the case of a relatively low RF-field amplitude $\sqrt{2} \cdot \omega_{1} \approx 0.3 \cdot \sigma_{E P R}$ (Figure $5(\mathrm{a}))$, the signal rises during the first $200 \mathrm{~ns}$ to approximately $30 \%$ of the final level. During the next $30 \mu \mathrm{s}$, the signal shows a slower exponential increase up to $90 \%$ of the final level. Afterwards the signal increases on the time scale of $110 \mu \mathrm{s}$, decays at times of about $350 \mu \mathrm{s}$, and stays constant afterwards. For higher RF-field amplitudes, e.g., $\sqrt{2} \cdot \omega_{1} \approx 0.7 \cdot \sigma_{E P R}$ (Figure 5(b)) the signal shows oscillations within the first $10 \mu \mathrm{s}$. Fourier analysis reveals two frequency components in these oscillations: the main frequency of $1.2 \mathrm{MHz}$ and a frequency component of about $3.4 \mathrm{MHz}$, which corresponds to the fast low-amplitude oscillations during the first $\mu \mathrm{s}$. After these oscillations are damped, the signal raises nearly exponential during $50 \mu$ s and stays constant afterwards. Figure 5(c) shows a measurement for the RF-field amplitude $\sqrt{2} \cdot \omega_{1} \approx 2.3 \cdot \sigma_{E P R}$, which is thus larger than $\sigma_{E P R}$. In this case, a fast and wellpronounced oscillation during the first $500 \mathrm{~ns}$ can be observed. The frequency of this oscillation is $11.5 \mathrm{MHz}$. After this
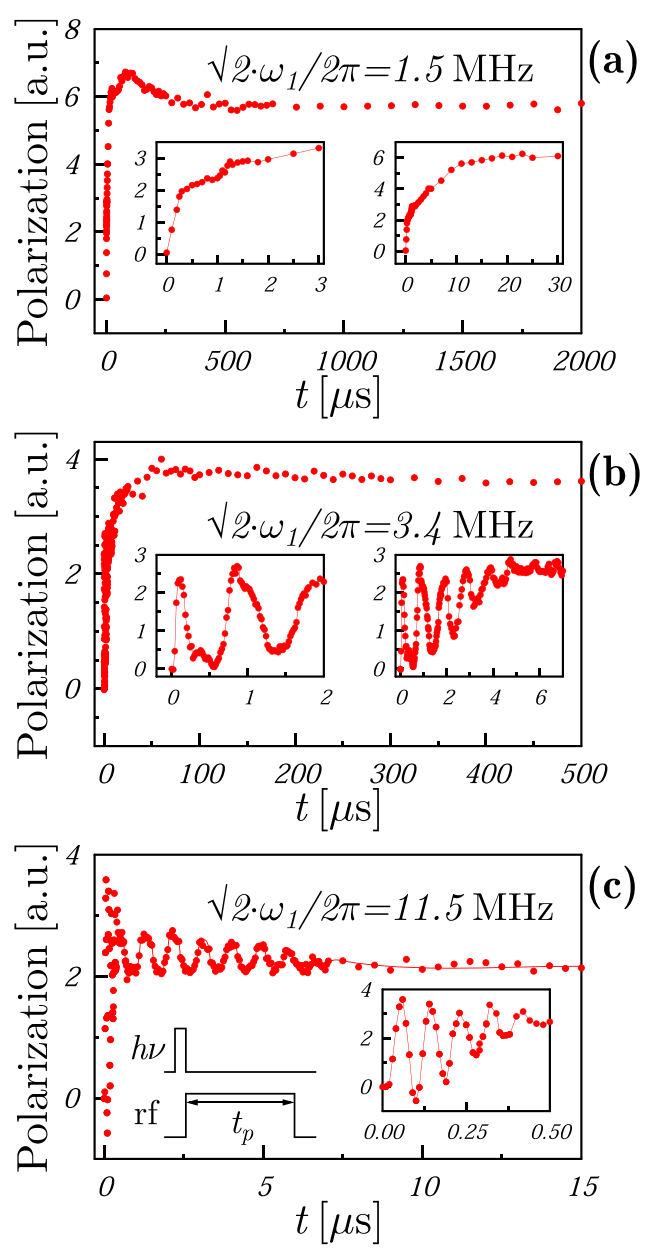

FIG. 5. Single pulse experiments for three different rf-amplitudes $\sqrt{2} \cdot \frac{\omega_{1}}{2 \pi}=1.5$ (a), 3.4 (b), and $11.5 \mathrm{MHz}$ (c). The time scales are different. The insets show the signal on a shorter time scale. (System Pe- $\mathrm{d}_{14} / \mathrm{Na}-\mathrm{h}_{8}$, $\frac{\omega_{\text {rf }}}{2 \pi}=230 \mathrm{MHz}=\frac{\omega_{0}}{2 \pi}+\frac{\sigma_{E P R}}{2 \pi}, \frac{\omega_{n}}{2 \pi}=1.2 \mathrm{MHz}, T=250 \mathrm{~K}$.) oscillation is damped, an oscillation with a slower frequency of $1.2 \mathrm{MHz}$ can be observed on a longer time scale of about $7 \mu \mathrm{s}$. Afterwards the signal stays constant.

These measurements clearly indicate that the polarization transfer dynamics shifts to a shorter time scale when $\omega_{1}$ is increased. Furthermore, one can observe well-pronounced oscillations, with two different frequencies when $\omega_{1}$ becomes comparable to or larger than $\sigma_{E P R}$. On the other hand, we see that the polarization transfer efficiency is maximal when the "matching condition" is fulfilled; on the other hand, pronounced oscillations are seen only when the spin system is away from this condition. The reason why at the "matching condition" the oscillations diminish will be discussed later. For demonstrating the coherent spin evolution we will from now on work somewhat away from the matching conditions. The spin dynamics in both cases is clarified in Sec. IV.

For investigating the correlation of the observed frequencies with experimental parameters, we have performed a series of experiments under systematic variation of several parameters, namely, the applied pulse sequence, the external field $B_{p o l}$, the $\omega_{1}$ amplitude, and the frequency offset $\Delta \omega$ relative to the center of the EPR-line, also varying the ONP crystal systems and their degree of deuteration. These experimental results are summarized in Figure 6. We denote the frequency of the fast oscillation (see, e.g., the inset of Figure 5(c)) by $\omega_{n u t}$, the nutation frequency, and the frequency of the slower oscillation by $\omega_{\text {pre }}$, the precession frequency. It turns out that only $\omega_{1}=\gamma_{e} \cdot B_{1}, \omega_{n}=\gamma_{n} \cdot B_{p o l}$, and $\Delta \omega$ have influence on the observed frequencies. All other parameters, in particular the applied pulse sequences and specific magnetic resonance parameters of the systems under investigation, have no effect on the frequencies.

The fast frequency is, as shown in Figure 6(a), for a frequency offset of $\Delta \omega \leq 2.5 \cdot \sigma_{E P R}$ directly proportional to $\omega_{1}$ where the proportionality constant is given by $\sqrt{2}$, i.e., $\omega_{\text {nut }}=\sqrt{2} \cdot \omega_{1}$. The frequency $\omega_{\text {nut }}$ was obtained by Fourier analysis of the observed oscillation, while $\omega_{1}$ was independently obtained by calibration of the $B_{1}$ field via the pulse length of $\pi$-NMR pulses as described in detail in Ref. 32. The dependence of $\omega_{\text {nut }}$ on the frequency offset is shown in Figure 6(b) in units of the EPR-line width. It turns out that $\omega_{\text {nut }}$ is given by $\sqrt{2} \cdot \omega_{1}$ for $\Delta \omega \leq 2.5 \cdot \sigma_{E P R}$ and by $\sqrt{2} \cdot \omega_{1}+\left(\Delta \omega-2.5 \cdot \sigma_{E P R}\right)$ for $\Delta \omega \geq 2.5 \cdot \sigma_{E P R}$. The slow frequency component, as shown in Figure 6(c), turns out to be determined solely by the external magnetic field, $\omega_{\text {pre }}=\omega_{n}=\gamma_{n} \cdot B_{p o l}$. The frequency $\omega_{\text {pre }}$ was determined by the Fourier analysis of the oscillations and $B_{p o l}$ was determined by detecting the nuclear transition frequencies at low field as described in Refs. 25, 50, and 51. The accuracy of determining the magnetic field was about $\pm 0.02 \mathrm{mT}( \pm 8 \mathrm{kHz})$.

Due to a similar dependence of the frequencies observed in magnetic resonance, we term the fast frequency component "nutation" of the electron spins and the slow component "precession" of the proton spins. For now, this is merely a nomenclature without any physical impact. However, our theoretical treatment given below shows that there is a true analogy between the commonly known nutations and precessions in magnetic resonance experiments and the oscillations found in 

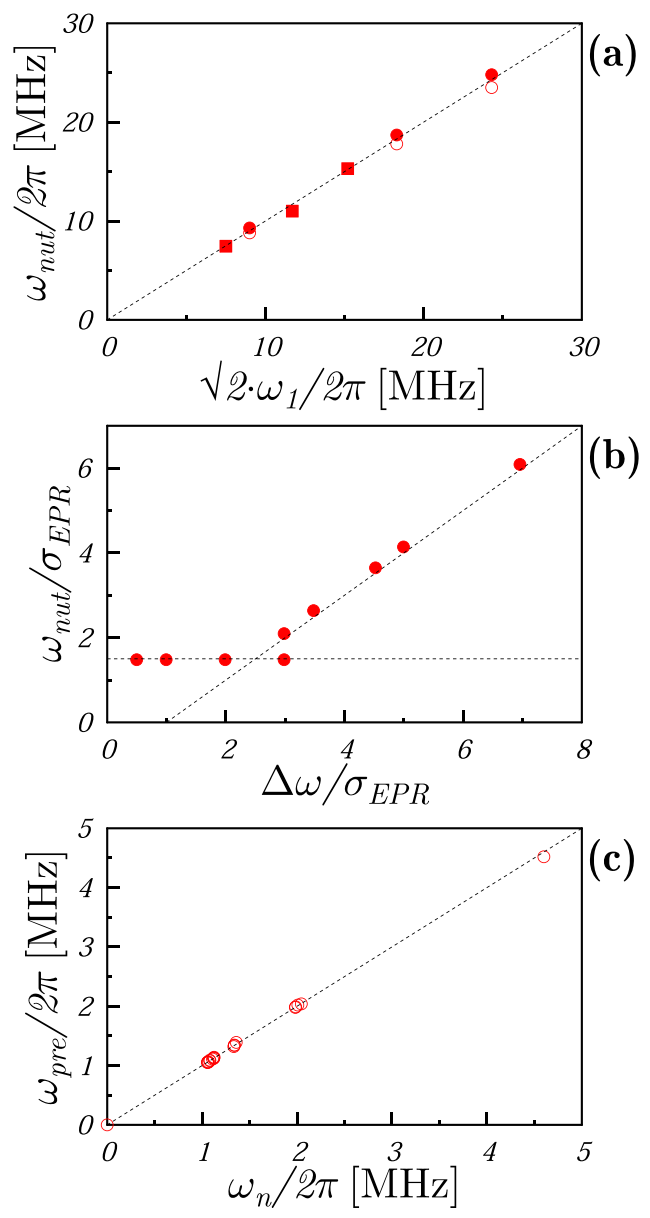

FIG. 6. Dependency of the observed frequency components on the experimental parameters. (a) The fast frequency component $\omega_{\text {nut }}$ in dependence of the RF-amplitude $\omega_{1}$ for different frequency offsets $\Delta \omega: \sigma_{E P R}=0$ (squares), 0.1 , (full circles) 1 (open circles). (b) The fast frequency component $\omega_{\text {nut }}$ in dependence of the frequency offset $\Delta \omega$ for a fixed value of $\omega_{1}$. (c) The slow frequency component $\omega_{\text {pre }}$ in dependence of the external magnetic field $\omega_{n}=\gamma_{n} \cdot B_{p o l}$. The experiments were performed on both systems and under various experimental conditions.

our experiments. The fact that $\omega_{\text {pre }} \approx \omega_{n}$ and $\omega_{\text {nut }} \approx \sqrt{2} \omega_{1}$ already gives a strong hint that this is indeed the case.

In Figure 7 two single-pulse experiments on the system Ac- $\mathrm{d}_{9} / \mathrm{Fl}-\mathrm{d}_{8} \mathrm{~h}_{2}$ are shown for two different external fields. At these experimental settings the nutations are strongly modulated by the precessions: the nutations and precessions interfere. The nutation frequency is however still given by $\sqrt{2} \cdot \omega_{1}$ and the modulation frequency is given by $\omega_{\text {pre }}=\omega_{n}$. At higher external fields (Figure 7(b)), the nutations and precessions are observable on different time scales. However, in this case, the nutations are damped on a faster time scale of about $200 \mathrm{~ns}$, and the precessions are damped on a time scale of $2 \mu \mathrm{s}$. At $\omega_{n}=4.2 \mathrm{MHz}$, an additional exponential increase with a time constant of $0.95 \mu$ s can be observed.

\section{Two-pulse sequences}

Single pulse experiments give non-zero ONP only when performed off-resonance, $\Delta \omega \neq 0$, as can be directly seen from Figure 2. Even then, the contributions of individual spin packets have the tendency to partly cancel each other, and only a reduced net signal can be obtained. Application of a pulse sequence at the center of the EPR-line can, in principle,
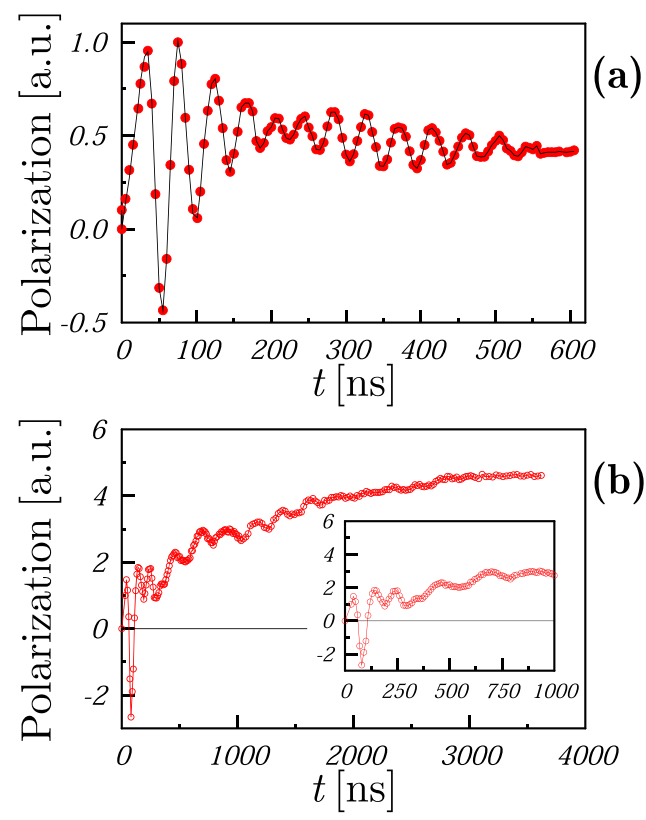

FIG. 7. Single pulse experiments on Ac- $\mathrm{d}_{9} / \mathrm{Fl}-\mathrm{d}_{8} \mathrm{~h}_{2}$ in two different external fields $\omega_{n}=\gamma_{n} \cdot B_{p o l}$. (a) $\frac{\omega_{r f}}{2 \pi}=181 \mathrm{MHz}=\frac{\omega_{0}}{2 \pi}+\frac{\sigma_{E P R}}{2 \pi}, \sqrt{2} \cdot \frac{\omega_{1}}{2 \pi}=23.5 \mathrm{MHz}$, $\frac{\omega_{n}}{2 \pi}=2.0 \mathrm{MHz}, T=300 \mathrm{~K}$. (b) $\frac{\omega_{r f}}{2 \pi}=1.5 \mathrm{GHz}=\frac{\omega_{0}}{2 \pi}+\frac{\sigma_{E P R}}{2 \pi}, \sqrt{2} \cdot \frac{\omega_{1}}{2 \pi}$ $=9.3 \mathrm{MHz}, \frac{\omega_{n}}{2 \pi}=4.2 \mathrm{MHz}, T=300 \mathrm{~K}$.

lead to a contribution of all spin packets without any cancellations, and hence to a higher nuclear polarization. Such a pulse sequence requires however a change of the symmetry of the Hamiltonian, which can be achieved in the simplest way by using at least two RF-pulses of different relative phase. Such experiments were first performed at high field, $\frac{\omega_{r f}}{2 \pi} \approx 9.5 \mathrm{GHz}$, $\frac{\omega_{n}}{2 \pi} \approx 16 \mathrm{MHz} \gg \frac{\sigma_{E P R}}{2 \pi} \mathrm{MHz} ;{ }^{31,45,52}$ however, in contrast to our experiments no pronounced oscillations have been found. For finding reasons for this different behavior and for establishing a common description, as well as for optimizing the achievable proton polarization, we have performed various multi-pulse experiments at low fields.

Figure 8 shows the dependency of the signal on the relative phase $\varphi$ of two pulses on resonance, i.e., $\Delta \omega=0$. The signal shows a dependency proportional to $\sin \varphi$ with a maximum

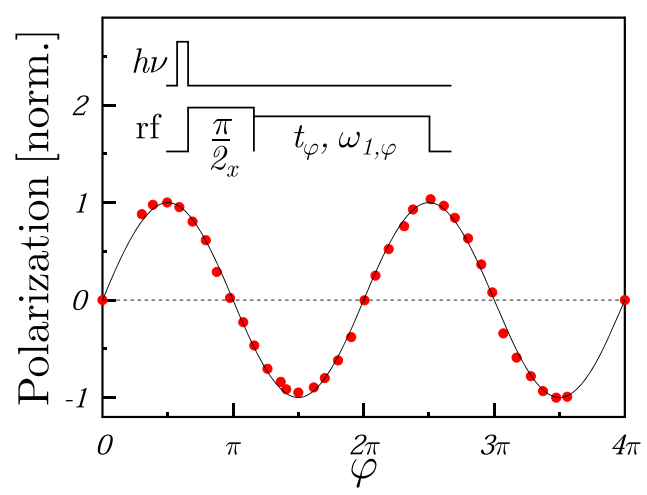

FIG. 8. Proton polarization as a function of the relative phase difference between two rf-pulses. The amplitude of the first $\left(\frac{\pi}{2}\right)_{x}$ pulse was $\sqrt{2} \cdot \frac{\omega_{1}}{2 \pi}$ $=12 \mathrm{MHz}$, and the amplitude of the second pulse was $\sqrt{2} \cdot \frac{\omega_{1}}{2 \pi}=4 \mathrm{MHz}$ with a length of $t_{\varphi}=9 \mu \mathrm{s}$. The solid line is $\operatorname{simply} \sin \varphi$. The applied pulse sequence is shown in upper part of the figure. (System Ac- $\mathrm{d}_{9} / \mathrm{Fl}-\mathrm{d}_{8} \mathrm{~h}_{2}, B_{p o l}=50 \mathrm{mT}$, $\frac{\omega_{r f}}{2 \pi}=213 \mathrm{MHz}=\frac{\omega_{0}}{2 \pi}, T=300 \mathrm{~K}$.) 
for $\varphi=\frac{\pi}{2}, \frac{3 \pi}{2}, \ldots$. This $\varphi$-dependency is independent of the length and amplitude of the applied pulses and is affected only by choice of $\Delta \omega$. Since the first maximum is reached at $\varphi=\frac{\pi}{2}$, we have performed our experiments with a phase shift of $\frac{\pi}{2}$ between the pulses.

The simplest sequence with a phase shift is a two-pulse sequence $t_{x}-t_{y}$. Figure 9 shows typical results of such experiments with different pulse lengths and amplitudes. The flip angle of the pulses is defined via the observed nutation frequency, e.g., a $\frac{\pi}{2}$ pulse corresponds to a pulse length of $t=\pi \cdot\left(2 \cdot \omega_{\text {nut }}\right)^{-1}$.

Heteronuclear cross-polarization under the HartmannHahn condition or adiabatic demagnetization in the rotating frame are commonly done employing a spin-lock sequence $\left(\frac{\pi}{2}\right)_{x}-t_{y}$. Figure 9 shows the result for such a sequence. To check whether such spin-locking plays a significant role in experiments of this kind, we have performed the experiment also with the inverted pulse sequence $\left(t_{y}-\left(\frac{\pi}{2}\right)_{x}\right)$. The signals are in both cases in qualitative agreement. On the short time scale, nutations are observable with a frequency given by the amplitude of the $t_{y}$ pulse multiplied by $\sqrt{2}$. On the longer time scale, precessions are observable with a frequency given by $\omega_{n}$. In contrast to the amplitude of the nutations, the amplitude of the precessions differs in both cases, but the maximum signal amplitude is the same in both cases. Whereas the oscillations are the same as for irradiation by a single off-resonant pulse, no constant final value (as in the case of, e.g., Figure 5(c)) can be observed. In comparison to the single-pulse experiments, the signal intensities in Figure 9 are about a factor of two greater under otherwise identical experimental conditions.

Our further experiments, see supplementary material, also revealed that polarization transfer exhibits qualitatively the same behavior also in the presence of spin-locking. Thus, upon varying the length of one of the pulses, the polarization transfer dynamics shows oscillations with two characteristic frequencies. One of these frequencies, $\omega_{\text {pre }}$, does not depend on the characteristics $\left(\omega_{1}, \Delta \omega\right)$ of the RF-pulses applied to the system. It is also independent of the local hyperfine fields and

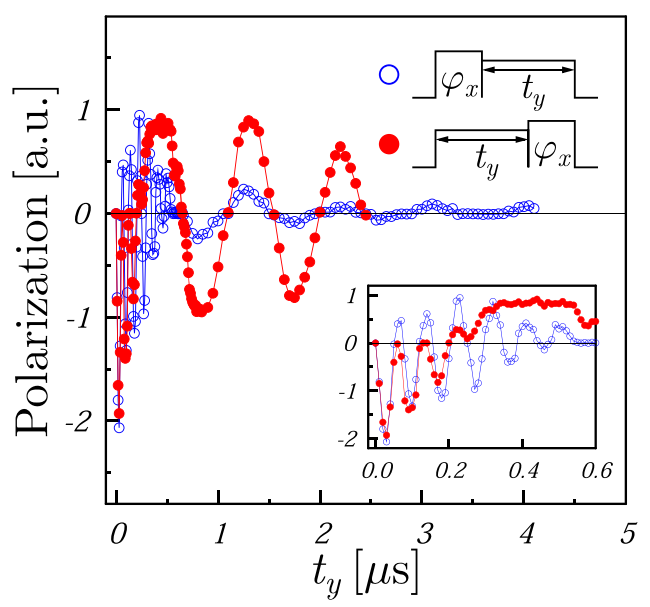

FIG. 9. Two pulse sequence with $\varphi=\frac{\pi}{2}$ phase shift, classical spin-lock sequence $\left(\frac{\pi}{2}\right)_{x}-t_{y}$, and inverted spin-lock sequence $t_{y}-\left(\frac{\pi}{2}\right)_{x}, \sqrt{2} \cdot \frac{\omega_{1 y}}{2 \pi}$ $=11.5$ (open circles), respectively, $12.0 \mathrm{MHz}$ (full circles). (System Pe$\mathrm{d}_{14} / \mathrm{Na}-\mathrm{h}_{8}, \frac{\omega_{r f}}{2 \pi}=230 \mathrm{MHz}=\frac{\omega_{0}}{2 \pi}, \frac{\omega_{n}}{2 \pi}=1.2 \mathrm{MHz}, T=250 \mathrm{~K}$.) depends only on the external magnetic field. Hence, switching off the RF-fields during certain time intervals might open an opportunity to investigate the motion of the spin system during free evolution periods where the dynamics of the spin system is determined only by the local fields and the static external field. Such experiments potentially give useful information about the mechanisms which lead to the precessions; the corresponding pulse sequences comprise a variable interpulse delay when no RF-excitation is used. Data obtained using such pulse sequences are shown in supplementary material; they demonstrate that these pulse sequences allow one to study precessions separately: the observed oscillation frequency is very close to $\omega_{n}$, and the ONP $\tau$-dependences hardly contain any contributions from the nutations.

\section{DISCUSSION}

\section{A. Summary of experimental observations}

The most conspicuous experimental finding of our experiments is the observation of oscillatory signal components in electron-nuclear polarization transfer. As is well known from other experiments in magnetic resonance, for instance, from $\operatorname{ESEEM}^{53}$ (electron spin echo envelope modulation) and pulsed ENDOR (electron nuclear double resonance) ${ }^{54}$ experiments, the appearance of such signal components is a clear indication for the involvement of spin coherences.

The ONP measurements include steps on well separated time scales. The optically induced electronic transitions take place within some $10 \mathrm{ps}$ and have no influence on the resulting nuclear polarization. During the subsequent RF-irradiation on the time scale of about $10 \mathrm{~ns}-10 \mu$ s the dynamics of the polarization transfer are observed. For triplet concentrations below $1000 \mathrm{ppm}$, the dipolar triplet-triplet interactions as well as the dipolar interaction among the nuclear spins are less than $20 \mathrm{kHz}$. The interactions among like spins are therefore effective only on the time scale of some $50 \mu$ s and can be neglected during the RF-irradiation. The experimental results of our measurements are therefore determined solely by the hyperfine interaction of the triplet with the nuclear spins in their surroundings. By using deuterated guest molecules and NMR detection of the proton polarization, we observe only polarization transfer to nearby protons of the matrix mediated by dipolar electron-nuclear interaction.

After the decay of the triplets, on the time scale of several $\mathrm{ms}$, the dipolar interaction among the nuclei is used to distribute the locally generated spin polarization over the whole matrix by spin diffusion. Since the deuterium and the proton Zeeman reservoirs are not on speaking terms, the deuterium nuclei do not participate in this process. After a few hundred polarization cycles, the external field is switched adiabatically during about $10 \mathrm{~s}$ to the detection field, where the achieved polarization is detected.

The observed oscillations of our experiments contain therefore information about the spin polarization transfer mechanisms. The experimental results about these oscillations can be summarized as follows:

(a) Two different frequency components are observable, which we denote nutations and precessions, respectively. 
(b) The nutation frequency $\omega_{\text {nut }}$ is determined solely by the RF-amplitude $\omega_{1}$ and by the frequency offset $\Delta \omega$. For small frequency off-sets, $\Delta \omega<2.5 \cdot \sigma_{E P R}$, the frequency is given by $\omega_{\text {nut }}=\sqrt{2} \cdot \omega_{1}$.

(c) The precession frequency $\omega_{\text {pre }}$ is determined only by $\omega_{n}: \omega_{\text {pre }}=\omega_{n}$.

(d) The frequency components have different characteristic damping time constants. The nutations are damped on a time scale of a few $100 \mathrm{~ns}$, whereas the precessions are damped on a time scale of several $\mu \mathrm{s}$. These damping times are approximately the same for all pulse sequences.

(e) The occurrence of these frequency components is independent of the applied pulse sequence and the systems under investigation.

(f) Only the relative amplitudes of the frequency components depend on the actual pulse sequence. Whereas both components are observable in experiments under variation of the length of one of the pulses, the nutations are, in contrast to the precessions, not observable in experiments with a variable RF-off time.

These results clearly indicate that these frequency components are a general feature of ONP polarization transfer being independent of the specific experimental settings. Hence, these results clearly necessitate developing a new model, which is presented below. We also briefly discuss the case $\omega_{\text {pre }} \approx \omega_{\text {nut }}$, in which the polarization transfer kinetics exhibits a different behavior.

By using different molecular systems, some additional properties of the oscillations were observed:

i. The triplet concentrations were varied in the range of $1-1000 \mathrm{ppm}$ without any observable changes in the experimental results. The frequency components are therefore independent of the triplet state concentration in this range.

ii. Since the lifetime of the triplet spin polarization is of different origin for both systems, the decay and relaxation mechanism within the triplet spin system have no influence on the frequency components.

In contrast to deuterated pentacene $\left(\mathrm{Pe}-\mathrm{d}_{14} / \mathrm{Na}-\mathrm{h}_{8}\right)$, it was not possible to observe oscillations in protonated pentacene (Pe$\mathrm{h}_{14} / \mathrm{Na}-\mathrm{h}_{8}$ ) for RF-amplitudes up to $\sqrt{2} \cdot \omega_{1}=20 \mathrm{MHz}$. This finding is in agreement with the results for Pe- $\mathrm{d}_{14} / \mathrm{Na}-\mathrm{h}_{8}$, indicating that the oscillations are only observable if $\sqrt{2} \cdot \omega_{1}$ is larger than or at least comparable to $\sigma_{E P R}$. We believe that the damping time constants of the nutations are directly determined by the $\sigma_{E P R}$. Hence, our current interpretation of this experimental finding is that only practical experimental restrictions (resulting from the limited maximal values of $\omega_{1}$ and from the achievable time resolution) do not allow us to observe the oscillations in Pe- $\mathrm{h}_{14} / \mathrm{Na}-\mathrm{h}_{8}$. This assumption is in agreement with results reported by Kouskov et al. ${ }^{55}$ In that work, electron-nuclear polarization transfer experiments have been performed on fully protonated systems at high external magnetic fields $\left(\omega_{n}=12 \mathrm{MHz}\right)$ and strong RF-fields $\left(\sqrt{2} \cdot \omega_{1}>\sigma_{E P R}\right)$. The authors observed transient oscillations with a frequency, which coincides with the nutation frequency in our experiments.
The total ONP achieved in our experiments is the same for fully protonated, partially protonated, and fully deuterated samples under otherwise identical experimental conditions, crystal geometries, and initial dopant concentrations in the melt. Hence-as argued above-intramolecular polarization transfer plays only a minor rule and the nuclear polarization observed in experiment comes from an intermolecular polarization transfer process.

The triplet spin concentration can be varied by changing the number of photons in the applied light flashes. In all our experiments, we found a linear dependency of the resulting nuclear polarization on the number of photons and consequently on the number of the triplet states created by light. With the total yield for the ISC of $15 \%,{ }^{56}$ a lower estimate of the quantum yield, i.e., the number of polarized protons per the excited triplet state, is about 0.07 for a single pulsed experiment shown in Figure 5(c) for the maximum of the signal in Pe- $\mathrm{d}_{14} / \mathrm{Na}-\mathrm{h}_{8}$. The quantum yield for $\mathrm{Ac}-\mathrm{d}_{9} / \mathrm{Fl}-\mathrm{d}_{8} \mathrm{~h}_{2}$ is of a comparable magnitude. ${ }^{57}$

As far as the polarization transfer efficiency is concerned, in all our experiments for $\sqrt{2} \cdot \omega_{1} \geq \sigma_{E P R}$ the maximum of the proton polarization is obtained during the oscillations. Usually the first maximum of the nutations corresponds to the highest ONP achievable. The dependence of the signal amplitude on $\omega_{1}$ in Figure 3 indicates that for pulses of this length a broad maximum exists. Due to the width of this maximum, the exact value of $\omega_{1}$ does not have to be set very precisely. However, values of $\sqrt{2} \cdot \omega_{1} \approx 12 \mathrm{MHz}$ require short pulses of the order of $20 \mathrm{~ns}$ to obtain the optimal signal. This short transfer time, was one of the main reasons to develop models which include at least two steps on different time scales, ${ }^{32,55}$ or rely on spinlocking of the electron spins. ${ }^{31}$

\section{B. Spin dynamics}

Here we provide only a simple semi-qualitative explanation of the experimentally observed effects. To do so, we use a simplified Hamiltonian of the triplet state. The simplification comes from the fact that we selectively drive EPR transitions only for two out of the three triplet sub-states. In this situation, a fictitious spin $1 / 2$, here denoted as $\hat{\boldsymbol{S}}$, can be assigned to this two-level system. ${ }^{29,58,59}$ Only the RF field strength $\omega_{1}$ has to be substituted by $\sqrt{2} \omega_{1}$. The reason is that when in a three-level system (i.e., in a triplet molecule) only one of the two transitions is excited the effective $B_{1}$-field needs to be redefined. ${ }^{58}$ The truncated electron-nuclear spin Hamiltonian in the RF-rotating frame (the frequency of rotation is $\omega_{r f}$ ) can be written as follows (written in the units of $\hbar$ ):

$$
\hat{H}_{r f}=\Delta \omega_{e} \hat{S}_{z}+\sqrt{2} \omega_{1} \hat{S}_{x}-\omega_{n} \hat{I}_{z}+A_{z z} \hat{S}_{z} \hat{I}_{z}+A_{z x} \hat{S}_{z} \hat{I}_{x},
$$

where $\Delta \omega=\left(\omega_{0}-\omega_{r f}\right)$ is the electron Zeeman interaction in the rotating frame with $\omega_{0}$ being the Zeeman interaction in the lab frame, $\omega_{n}$ is the nuclear Zeeman interaction, $A_{z z}$ and $A_{z x}$ are the secular and pseudo-secular parts of the hyperfine coupling. In general, the hyperfine tensor has more components, but in the high-field approximation (i.e., when $\omega_{0}$ is much greater than the hyperfine coupling) only these two terms should be maintained, whereas other hyperfine terms can be neglected. In ONP keeping the $A_{z x}$-term is of basic importance: isotropic 
hyperfine interaction itself cannot drive polarization transfer in our experiments.

The initial state of the electron-nuclear spin system is characterized by a non-zero electronic polarization, which, in turn, is proportional to the population difference of the corresponding triplet sublevels. The electron spin polarization vector is usually parallel to the $z$-axis, i.e., to the external magnetic field. Nuclear polarization at $t=0$ is taken negligibly small.

Calculations of the spin dynamics using the Hamiltonian $\hat{H}_{r f}$ are relatively complex because the Hamiltonian written in matrix form cannot be easily split into $2 \times 2$ blocks. To go on further, let us "tilt" the reference frame for the electron spin, so that the electronic Zeeman term is simplified as follows:

$$
\Delta \omega_{e} \hat{S}_{z}+\sqrt{2} \omega_{1} \hat{S}_{x}=\omega_{e}^{\prime} \hat{S}_{z}^{\prime}
$$

where $\omega_{e}^{\prime}=\sqrt{\Delta \omega_{e}^{2}+2 \omega_{1}^{2}}$. That is, the $z$-axis of the new frame is parallel to the effective field vector $\omega=\left(\omega_{1}, 0, \Delta \omega_{e}\right)$ in the original rotating frame. Schematic representation of the titled frame is given in Scheme 1. In the tilted frame the Hamiltonian is modified,

$$
\begin{aligned}
\hat{H}_{r f}^{\prime}= & \omega_{e}^{\prime} \hat{S}^{\prime}-\omega_{n} \hat{I}_{z}+A_{z z} \cos \theta \hat{S}_{z}^{\prime} \hat{I}_{z}-A_{z z} \sin \theta \hat{S}_{x}^{\prime} \hat{I}_{z} \\
& +A_{z x} \cos \theta \hat{S}_{z}^{\prime} \hat{I}_{x}-A_{z x} \sin \theta \hat{S}_{x}^{\prime} \hat{I}_{x} .
\end{aligned}
$$

Thus, the electronic Zeeman interaction is simplified in the new frame as the field vector is parallel to the new $z$-axis (hereafter $z^{\prime}$ ), but new hyperfine terms appear. When the relation between the Zeeman interactions and $A_{z z}, A_{z x}$ is arbitrary, the Hamiltonian cannot be easily solved. However, when $\omega_{e}^{\prime}, \omega_{n} \gg A_{z z}, A_{z x}$ the zero-order eigen-states of $\hat{H}_{r f}^{\prime}$ are characterized by the projections of the spins on their field axes, which are $z^{\prime}$ for the electron spins and $z$ for the nuclear spins. This means that the electronic and nuclear spins precess about the corresponding field vectors $\omega^{\prime}{ }_{e}$ and $\omega_{n}$, respectively. Hyperfine interactions can be treated as a perturbation, which leads to exchange of polarization between electrons and nuclei. Let us consider such a polarization transfer. For simplicity, we use the Hamiltonian valid for $\theta=\frac{\pi}{2}$ and $A_{z z}=0$,

$$
\hat{H}_{r f}^{\prime} \rightarrow \omega_{e}^{\prime} \hat{S}_{z}^{\prime}-\omega_{n} \hat{I}_{z}-A_{z x} \hat{S}_{x}^{\prime} \hat{I}_{x} .
$$

Thus, there is only one hyperfine term left. The structure of this term is as follows:

$$
\hat{S}_{x}^{\prime} \hat{I}_{x}=\frac{\hat{S}_{+}^{\prime} \hat{I}_{-}+\hat{S}_{-}^{\prime} \hat{I}_{+}}{4}+\frac{\hat{S}_{+}^{\prime} \hat{I}_{+}+\hat{S}_{-}^{\prime} \hat{I}_{-}}{4} .
$$
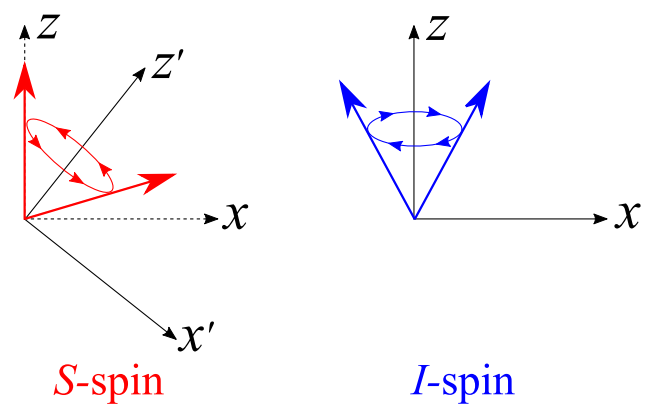

I-spin

SCHEME 1. Tilted frame: the electron is precessing about the effective field $\omega_{e}^{\prime}|| z^{\prime}$ (left) while the nucleus is precessing about the $z$-axis of the lab frame (right).
Thus, the perturbation drives transitions, which flip both spins; such transitions are either zero-quantum (i.e., the two spins flip, remaining anti-parallel) or double-quantum (i.e., the two spins flip, remaining parallel). The Hamiltonian matrix is as follows:

$$
\hat{H}_{r f}^{\prime}=\left(\begin{array}{cccc}
\Delta / 2 & 0 & 0 & V \\
0 & \Sigma / 2 & V & 0 \\
0 & V & -\Sigma / 2 & 0 \\
V & 0 & 0 & -\Delta / 2
\end{array}\right),
$$

where $\Delta=\left(\omega_{e}^{\prime}-\omega_{n}\right), \Sigma=\left(\omega_{e}^{\prime}+\omega_{n}\right), V=A_{z x} / 4$ comes from the pseudo-secular hyperfine coupling. The basis that we use is as follows: $\left|\alpha^{\prime} \alpha_{N}\right\rangle,\left|\alpha^{\prime} \beta_{N}\right\rangle,\left|\beta^{\prime} \alpha_{N}\right\rangle,\left|\beta^{\prime} \beta_{N}\right\rangle$. As usual, $|\alpha\rangle$ and $|\beta\rangle$ stand for spin-1/2 parallel and anti-parallel to the quantization axis; here the prime means that this axis is $z^{\prime}$ for the electron spin. The simplified and truncated Hamiltonian can be solved analytically because its splits into two $2 \times 2$ blocks (spanned by the states $\alpha^{\prime} \alpha_{N}, \beta^{\prime} \beta_{N}$ and $\left.\alpha^{\prime} \beta_{N}, \beta^{\prime} \alpha_{N}\right)$. Knowing the initial density matrix, $\rho_{0}$, of the spin system we can calculate the density matrix, $\rho(t)$, at later times in the usual way (given by the general solution of the Liouville-von Neumann equation with a time-independent Hamiltonian),

$$
\rho(t)=\exp \left(-i \hat{H}_{r f}^{\prime} t\right) \rho_{0} \exp \left(i \hat{H}_{r f}^{\prime} t\right) .
$$

The expectation value of any spin operator $\hat{A}$ at the instant of time $t$ can be calculated as $\langle A\rangle(t)=\operatorname{Tr}\{\hat{A} \rho(t)\}$. When assuming that initially the electron spin has unity polarization parallel to the $z^{\prime}$-axis, $S_{z}^{\prime}(t=0)=1$, we obtain the following result:

$$
\begin{aligned}
\left\langle I_{z}\right\rangle(t)= & V^{2}\left\{\frac{\cos \sqrt{4 V^{2}+\Delta^{2}} t}{4 V^{2}+\Delta^{2}}-\frac{\cos \sqrt{4 V^{2}+\Sigma^{2}} t}{4 V^{2}+\Sigma^{2}}\right. \\
& \left.-\frac{\left(\Sigma^{2}-\Delta^{2}\right)}{\left(4 V^{2}+\Delta^{2}\right)\left(4 V^{2}+\Sigma^{2}\right)}\right\},\left\langle S_{z}^{\prime}\right\rangle(t)=1-I_{z}(t) .
\end{aligned}
$$

Thus, there are quantum oscillations with two frequencies: $\omega_{\Delta}=\sqrt{4 V^{2}+\Delta^{2}}$ and $\omega_{\Sigma}=\sqrt{4 V^{2}+\Sigma^{2}}$. The behavior of the polarization depends on the relation between the coupling matrix element, $V$, and the energy gaps, $\Delta$ and $\Sigma$.

When $V$ is much greater than $\Delta$ or $\Sigma$ the corresponding frequency, $\omega_{\Delta}$ or $\omega_{\Sigma}$, becomes minimal and tends to $2|V|=\left|A_{z x}\right| / 2$; at the same time the amplitude of the corresponding cosine function is maximal and tends to 1 . However, this situation is not favorable for observing the quantum oscillations, once there are several nuclei present with different hyperfine couplings: the time trace becomes a superposition of cosines oscillating at different frequencies with the consequence that the oscillations are rapidly decaying due to the spread of the $V$ values. This is consistent with the data shown in Figure 5(a).

In the case where $|V| \ll \Delta, \Sigma$, the time dependence of polarization can be greatly simplified because $\omega_{\Delta} \approx \omega_{e}^{\prime}-\omega_{N}$ and $\omega_{\Sigma} \approx \omega_{e}^{\prime}+\omega_{n} ; 4 V^{2}$ can also be neglected in the numerators in the expression for $\left\langle I_{z}\right\rangle(t)$. After some calculations, we obtain 


$$
\begin{aligned}
\left\langle I_{z}\right\rangle(t)= & \frac{2 V^{2}}{\left(\omega_{e}^{\prime}\right)^{4}}\left\{2 \omega_{e}^{\prime} \omega_{n} \cos \omega_{e}^{\prime} t \cos \omega_{n} t\right. \\
& \left.-\left(\left(\omega_{e}^{\prime}\right)^{2}-\omega_{n}^{2}\right) \sin \omega_{e}^{\prime} t \sin \omega_{n} t-2 \omega_{e}^{\prime} \omega_{n}\right\} .
\end{aligned}
$$

Thus, we have oscillatory components with the frequencies $\omega_{e}^{\prime}$ and $\omega_{n}$, that is, our simplified model agrees with the experimental observations. The evolution frequencies $\omega_{e}^{\prime}$ and $\omega_{n}$ are indeed equal to the "nutation" frequency of the electron spin (i.e., to $\sqrt{\Delta \omega_{e}^{2}+2 \omega_{1}^{2}}$ ) and the "precession" frequency of the nuclear spin (i.e., to $\omega_{n}$ ), which is consistent with our experimental findings.

The presence of further hyperfine coupling terms is expected to change the amplitude of the resulting nuclear polarization in the case $|V| \ll \Delta, \Sigma$, but electronic "nutations" and nuclear "precessions" should remain the dominant oscillatory contributions. When $A_{z z}$ and $A_{z x}$ are large the effects of these residual terms are expected to be stronger; however, here we are not going to discuss such effects in detail (such a discussion would require systematic numerical analysis of the $\left\langle I_{z}\right\rangle(t)$ time traces).

There is still one more point that can be addressed with our simple treatment. The size of the polarization transferred to nuclear spins is proportional to the initial value of $\left\langle S_{z}^{\prime}\right\rangle$ but not $\left\langle S_{z}\right\rangle$. Both quantities are related via the tilt angle $\theta$,

$$
\left\langle S_{z}^{\prime}\right\rangle=\cos \theta\left\langle S_{z}\right\rangle
$$

Thus, we obtain $\left\langle S_{z}^{\prime}\right\rangle=0$ when $\theta=\frac{\pi}{2}$. This means that for the transfer by a single RF-pulse, one should not pump the electron spin exactly at resonance, i.e., $\Delta \omega$ should be non-zero. Likewise, when the EPR line is inhomogeneously broadened and symmetric, one should not pump at the center of the line. Indeed, components with the same absolute value but of opposite sign of $\Delta \omega$ would yield initial $\left\langle S_{z}^{\prime}\right\rangle$ values, which exactly compensate each other. A better solution is to step out of resonance such that components with positive $\Delta \omega$ dominate over those with negative $\Delta \omega$ (or vice versa). An even better solution is provided by a two-pulse experiment: the first pulse converts $\left\langle S_{z}\right\rangle$ into $\left\langle S_{x}\right\rangle$. Since for resonant excitation $(\Delta \omega=0)$ the $x$-axis coincides with the $z^{\prime}$-axis of the tilted frame, we start with $\left\langle S_{z}^{\prime}\right\rangle=1$ at $t=0$ providing optimal conditions for polarization transfer. This is the essence of the Nuclear Orientation Via Electron spin Locking (NOVEL) method. ${ }^{27}$

Another way ${ }^{29}$ of describing the spin dynamics in ONP is based on using the product operator formalism. ${ }^{60,61}$ This method does not immediately provide the frequencies of oscillations of polarization, but it can clearly identify the pathway of polarization transfer and interactions, which are responsible for ONP formation. According to the product operator formalism we present the density matrix as a sum of basis spin operators, $\hat{B}$. The operators represent a full basis and are normalized, i.e., $\operatorname{Tr}\left\{\hat{B}_{k} \hat{B}_{l}\right\}=\delta_{k l}$. For an $\{S, I\}$ two-spin system, these operators are ${ }^{60,61}$ the unity operator $\hat{E} / 2$, components of the $\hat{\boldsymbol{S}}$ operator (i.e., $\hat{S}_{x}, \hat{S}_{y}, \hat{S}_{z}$ ), components of the $\hat{\boldsymbol{I}}$ operator (i.e., $\hat{I}_{x}, \hat{I}_{y}, \hat{I}_{z}$ ), and all products containing two operators, i.e., $2 \hat{S}_{m} \hat{I}_{n}$ (where $m, n=x, y, z$ ). The expression for $\rho$ is then as follows:

$$
\rho=\sum_{k} b_{k}(t) \hat{B}_{k}, b_{k}(t)=\operatorname{Tr}\left\{\sigma(t) \hat{B}_{k}\right\} .
$$

Substituting this expression into the Liouville-von Neumann equation for the density matrix we obtain that the operators $b_{k}(t) \hat{B}_{k}$ form sub-sets, which are coupled. In the absence of RF-excitation, there are three such subsets; see Scheme 2,

$$
\begin{gathered}
\text { (I) } \hat{S}_{z} \text {; (II) } \hat{S}_{x}, \hat{S}_{y}, 2 \hat{S}_{x} \hat{I}_{x}, 2 \hat{S}_{x} \hat{I}_{y}, 2 \hat{S}_{x} \hat{I}_{z}, 2 \hat{S}_{y} \hat{I}_{x}, 2 \hat{S}_{y} \hat{I}_{y}, 2 \hat{S}_{y} \hat{I}_{z} \text {; } \\
\text { (III) } 2 \hat{S}_{z} \hat{I}_{x}, 2 \hat{S}_{z} \hat{I}_{y}, 2 \hat{S}_{z} \hat{I}_{z}, \hat{I}_{x}, \hat{I}_{y}, \hat{I}_{z} .
\end{gathered}
$$

Within each sub-set the operators are mixed by the secular and pseudo-secular hyperfine interaction, as well as by the Zeeman interactions of the two spins with the external magnetic field. In ONP our goal is to transfer the electron spin polarization, represented by the $\hat{S}_{z}$ operator, to the nuclear spin; the corresponding polarization is given by the $\hat{I}_{z}$ operator. In the absence of RF-excitation these operators are belonging to different subsets, i.e., they are not mixed. The situation changes, when RF-excitation is turned on, which induces coupling (I) $\leftrightarrow$ (II) and (II) $\leftrightarrow$ (III), see Scheme 2 . As a result, starting from $\hat{S}_{z}$ one can deliver the spin order to the target spin order $\hat{I}_{z}$. One can also see that the polarization transfer pathway $\hat{S}_{z} \rightarrow \hat{I}_{z}$ becomes disconnected (in subset (III)) when the pseudo-secular hyperfine coupling is turned off. Thus, for RF-induced ONP, it is

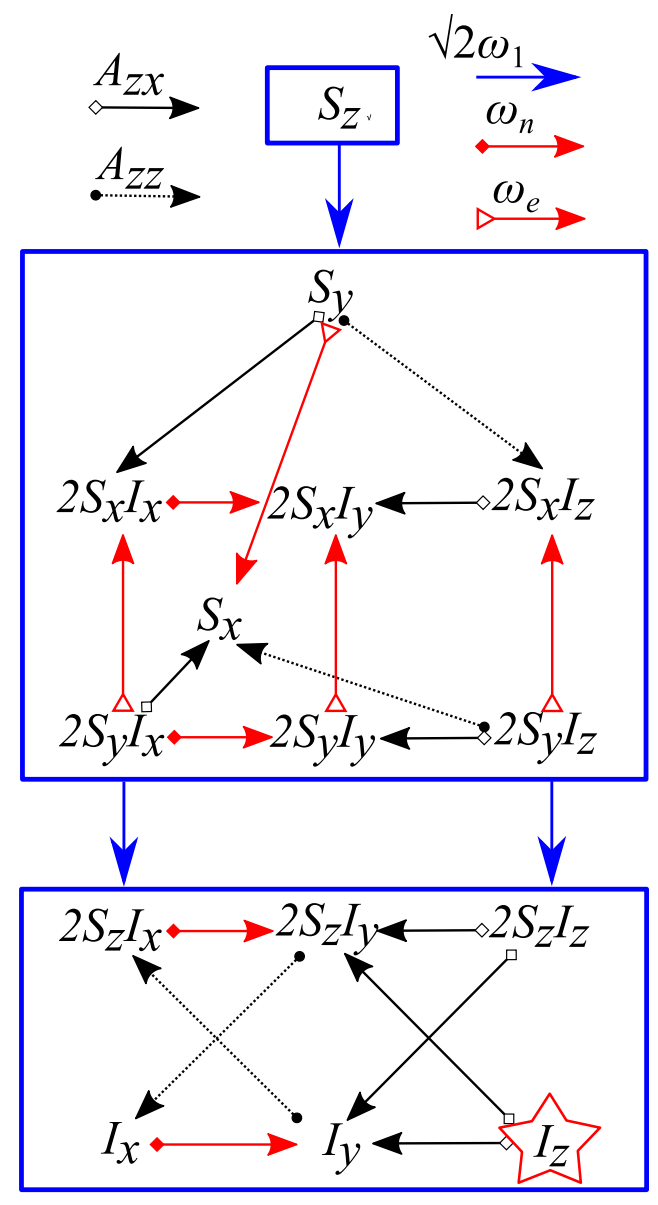

SCHEME. 2. Graphical representation of the product operators and their evolution. Subsets, disconnected in the absence of RF-pumping, are indicated by rectangular frames. The target spin order, $\hat{I}_{z}$, is indicated. Different styles of arrow correspond to different interaction terms in the Hamiltonian (as indicated in the scheme). See text for discussion. 
crucial that there are non-averaged anisotropic interactions present: isotropic hyperfine coupling alone cannot provide the desired polarization transfer pathway and the ONP effect itself. The presence of the secular hyperfine interaction changes the resulting polarization, since the transfer pathways of interest also involve the corresponding term in the Hamiltonian, but ONP can be formed even when $A_{z z}=0$.

\section{CONCLUSIONS}

ONP experiments with high temporal resolution enable a detailed study and precise manipulation of the electronnuclear polarization transfer. Light-induced spin-polarized triplet states at low concentrations (1-1000 ppm) serve as good model systems of a highly ordered isolated $S$ spin coupled to a limited number of $I$ spins to be polarized. Here, the interpretation of the experimental results is dramatically simplified allowing the full transfer dynamics to be mapped out. The experiments show oscillations with two characteristic frequency components, which are termed electronic spin "nutations" and nuclear spin "precessions." This tentative assignment of the two types of spin motion stems from the fact that the "nutation" frequency is close to $\sqrt{2} \cdot \omega_{1}$ and the "precession" frequency is close to $\omega_{n}$. As we also show in this work such an assignment indeed agrees with the specific kind of coherent spin motion that is named spin nutation ${ }^{62}$ and precession. These frequency components should be regarded as a general feature of the polarization transfer itself.

ONP represents an ideal case for probing electron-nuclear polarization transfer, since (i) the resulting ONP is very high enabling sensitive NMR detection and (ii) the spin system is prepared by light excitation in a well-defined state, suitable for subsequent manipulations. This allowed us to show that the electron-nuclear polarization transfer in ONP relies on coherent spin motion; a clear manifestation of this fact is the presence of oscillatory components in the ONP time dependence. Electron-nuclear spin coherences can also be excited in various EPR experiments; prominent examples are given by ESEEM and pulsed ENDOR experiments. Generally, coherent polarization transfer is much faster and by far more efficient than cross-relaxation based transfer and, therefore, advantageous for many applications. An important aspect of coherent transfer is the precise control and manipulation of rapidly oscillating spin coherences for creating the maximal nuclear spin polarization. In our case, this is reached by applying short RFpulses and using various RF-excitation schemes, which are discussed in detail.

Correct interpretation of the reported experimental results also stimulates us to develop an appropriate theoretical description of coherent spin dynamics in ONP. This will be done separately; specifically, we aim to elaborate a common description for polarization transfer under the action of RFexcitation and at level crossings in the electronic triplet subsystem. We anticipate that such a treatment will allow us to cover a wide range of experiments and provide their quantitative description. Such a treatment is inspired by the theoretical description of CIDNP (recently developed by some of us). ${ }^{63}$ The idea of this method is based on the analysis of the spin dynamics at level crossings. ${ }^{64}$
Finally, it is of interest to extend the present methodology from molecular crystals, where there is a single fixed orientation of the spin interaction tensors, to disordered media. In this situation, ONP could be used as a general tool to spinpolarize various matrices containing molecules of interest, e.g., biomolecules. Moreover, ONP can be used as an alternative to the popular DNP method. Indeed, ONP does not require low temperatures and starts with almost $100 \%$ electronic polarization; furthermore, the paramagnetic species are short-lived and thus disturb neither the NMR lineshape, nor nuclear spin diffusion processes. For the same reason, their effect on relaxation is minor. Experiments by Tateishi et al. ${ }^{65}$ show that ONP is this case is feasible. Such a development of ONP techniques can therefore provide a powerful resource for spin hyperpolarization in various solid-state NMR experiments.

\section{SUPPLEMENTARY MATERIAL}

See supplementary material for description of the experimental setup and additional measurements.

\section{ACKNOWLEDGMENTS}

This research has been supported by the Russian Science Foundation (Project No. 15-13-20035). G.B. gratefully acknowledges financial support by the Deutsche Forschungsgemeinschaft (Grant No. Bu-911-26/1). Our study would not have been possible without the experimental data provided by Dr. Martin Benkert. Also, Bartholomäus Heusinger contributed with data from his Diploma Thesis.

${ }^{1}$ T. Fujiwara and A. Ramamoorthy, "How far can the sensitivity of NMR be increased?," in Annual Reports on NMR Spectroscopy, edited by G. A. Webb (Academic Press, 2006), Vol. 58, p. 155.

${ }^{2}$ A. W. Overhauser, Phys. Rev. 92, 411 (1953).

${ }^{3}$ A. Abragam and M. Goldman, Rep. Prog. Phys. 41, 395 (1978).

${ }^{4}$ V. A. Atsarkin, Sov. Phys. Usp. 21, 725 (1978).

${ }^{5}$ T. Maly, G. T. Debelouchina, V. S. Bajaj, K.-N. Hu, C.-G. Joo, M. L. MakJurkauskas, J. R. Sirigiri, P. C. A. van der Wel, J. Herzfeld, R. J. Temkin, and R. G. Griffin, J. Chem. Phys. 128, 052211 (2008).

${ }^{6}$ G. Maier and H. C. Wolf, Z. Naturforsch. A 23, 1068 (1968).

${ }^{7}$ B. M. Goodson, Annu. Rep. NMR Spectrosc. 55, 299 (2005).

${ }^{8}$ D. Stehlik, "The mechanism of optical nuclear polarization in solids," in Excited States, edited by E. C. Lim (Academic Press, 1977), Vol. 3, p. 203.

${ }^{9}$ G. Lampel, Phys. Rev. Lett. 20, 491 (1968).

${ }^{10}$ R. Tycko, S. E. Barrett, G. Dabbagh, L. N. Pfeiffer, and K. W. West, Science 268, 1460 (1995).

${ }^{11}$ E. C. Reynhardt and G. L. High, J. Chem. Phys. 109, 4090 (1998).

${ }^{12}$ R. Fischer, C. O. Bretschneider, P. London, D. Budker, D. Gershoni, and L. Frydman, Phys. Rev. Lett. 111, 057601 (2013).

${ }^{13}$ H. W. van Kesteren, W. T. Wenckebach, J. Schmidt, and N. J. Poulis, Chem. Phys. Lett. 89, 67 (1982).

${ }^{14}$ T. R. Eichhorn, M. Haag, B. van den Brandt, P. Hautle, and W. T. Wenckebach, Chem. Phys. Lett. 555, 296 (2013).

${ }^{15}$ K. M. Salikhov, Y. N. Molin, R. Z. Sagdeev, and A. L. Buchachenko, Spin Polarization and Magnetic Effects in Radical Reactions (Elsevier, Amsterdam, 1984).

${ }^{16}$ W. Köckenberger, eMagRes 3, 161 (2014).

${ }^{17}$ K. Tateishi, M. Negoro, S. Nishida, A. Kagawa, Y. Morita, and M. Kitagawa, Proc. Natl. Acad. Sci. U. S. A. 111, 7527 (2014).

${ }^{18}$ K. H. Hausser and D. Stehlik, "Dynamic nuclear polarization in liquids," in Advances in Magnetic Resonance, edited by J. S. Waugh (Academic, New York, 1968), Vol. 3, p. 79.

${ }^{19}$ J. H. Noggle and R. E. Schirmer, The Nuclear Overhauser Effect: Chemical Applications (Academic Press, New York and London, 1971).

${ }^{20}$ C. D. Jeffries, Phys. Rev. 106, 164 (1957).

${ }^{21}$ Y. Hovav, A. Feintuch, and S. Vega, J. Magn. Reson. 207, 176 (2010). 
${ }^{22}$ C. F. Hwang and D. A. Hill, Phys. Rev. Lett. 19, 1011 (1967).

${ }^{23}$ Y. Hovav, A. Feintuch, and S. Vega, J. Magn. Reson. 214, 29 (2012).

${ }^{24}$ J. P. Colpa and D. Stehlik, Chem. Phys. 21, 273 (1977).

${ }^{25}$ J. P. Colpa, D. Stehlik, and H. M. Vieth, Chem. Phys. 47, 73 (1980).

${ }^{26}$ A. Henstra, P. Derksen, and W. T. Wenckebach, Phys. Lett. A 134, 134 (1988).

${ }^{27}$ A. Henstra and W. T. Wenckebach, Mol. Phys. 106, 859 (2008).

${ }^{28}$ J. P. Colpa, K. H. Hausser, and D. Stehlik, Z. Naturforsch., A 26, 1792 (1971).

${ }^{29}$ M. Benkert and H.-M. Vieth, Chem. Phys. Lett. 230, 153 (1994).

${ }^{30}$ V. Macho, D. Stehlik, and H.-M. Vieth, Chem. Phys. Lett. 180, 398 (1991).

${ }^{31}$ D. J. van den Heuvel, A. Henstra, T.-S. Lin, J. Schmidt, and W. T. Wenckebach, Chem. Phys. Lett. 188, 194 (1992).

${ }^{32}$ G. Buntkowsky, D. Stehlik, H.-M. Vieth, and K. M. Salikhov, J. Phys.: Condens. Matter 3, 6093 (1991).

${ }^{33}$ N. Bloembergen, Physica 15, 386 (1949).

${ }^{34}$ A. G. Redfield, Phys. Rev. B 116, 315 (1959).

${ }^{35}$ M. Ernst and B. H. Meier, "Spin diffusion in solids," Studies in Physical and Theoretical Chemistry (Elsevier, New York, 1998), Vol. 84, pp. 83-121.

${ }^{36}$ C. Ramanathan, Appl. Magn. Reson. 34, 409 (2008).

${ }^{37}$ Y. Hovav, A. Feintuch, and S. Vega, J. Chem. Phys. 134, 074509 (2011).

${ }^{38}$ S. C. Abrahams, J. M. Robertson, and J. G. White, Acta Crystallogr. 2, 233 (1949).

${ }^{39}$ W. H. Hesselink and D. A. Wiersma, J. Chem. Phys. 74, 886 (1981).

${ }^{40}$ D. E. Cooper, R. W. Olson, and M. D. Fayer, J. Chem. Phys. 72, 2332 (1980).

${ }^{41}$ W. H. Hesselink and D. A. Wiersma, J. Chem. Phys. 73, 648 (1980).

${ }^{42}$ C. A. Hutchison, Jr. and G. A. Pearson, J. Chem. Phys. 47, 520 (1967).

${ }^{43}$ T.-S. T. Lin, J.-L. Ong, D. J. Sloop, and H.-L. Yu, Pulsed EPR: A New Field of Applications (North-Holland Publishing Co., Amsterdam, 1989).

${ }^{44}$ J.-L. Ong, D. J. Sloop, and T.-S. Lin, J. Phys. Chem. 97, 7833 (1993).

${ }^{45}$ R. H. Fritsch, H. Brunner, and K. H. Hausser, Chem. Phys. 151, 261 (1991).

${ }^{46}$ H. Seidel, M. Mehring, and D. Stehlik, Chem. Phys. Lett. 104, 552 (1984).
${ }^{47}$ J.-L. Ong, D. J. Sloop, and T.-S. Lin, Chem. Phys. Lett. 241, 540 (1995).

${ }^{48}$ A. J. van Strien and J. Schmidt, Chem. Phys. Lett. 70, 513 (1980).

${ }^{49}$ H. Kolb and H. C. Wolf, Z. Naturforsch. A 27, 51 (1972).

${ }^{50}$ H.-M. Vieth, V. Macho, and D. Stehlik, Chem. Phys. Lett. 60, 368368 (1979).

${ }^{51}$ H.-M. Vieth, V. Macho, and D. Stehlik, J. Phys. Chem. 83, 3435 (1979).

${ }^{52}$ D. J. van den Heuvel, J. Schmidt, and W. T. Wenckebach, Chem. Phys. 187, 365 (1994).

${ }^{53}$ L. G. Rowan, E. L. Hahn, and W. B. Mims, Phys. Rev. 137, A61 (1965).

${ }^{54}$ H. Thomann and M. Bernardo, Isr. J. Chem. 32, 323 (1992).

${ }^{55}$ V. Kouskov, D. J. Sloop, S.-B. Lin, and T.-S. Lin, J. Magn. Reson. A 117, 9 (1995).

${ }^{56}$ W. H. Hesselink and D. A. Wiersma, "Theory and experimental aspects of photon echoes in molecular solids," in Modern Problems in Condensed Matter Sciences (North-Holland Publishing Co., Amsterdam, 1983), Vol. 4.

${ }^{57}$ D. Stehlik and H.-M. Vieth, "Time evolution of electron-nuclear crosspolarization in radiofrequency induced optical nuclear polarization (RFONP)," in Pulsed Magnetic Resonance: NMR, ESR, and Optics, edited by D. M. S. Bagguley (Clarendon Press, Oxford, 1992).

${ }^{58}$ S. Vega and A. Pines, J. Chem. Phys. 66, 5624 (1977).

${ }^{59}$ R. P. Feynman, F. L. Vernon, and R. W. Hellwarth, J. Appl. Phys. 28, 49 (1957).

${ }^{60}$ R. R. Ernst, G. Bodenhausen, and A. Wokaun, Principles of Nuclear Magnetic Resonance in One and Two Dimensions (Clarendon Press, Oxford, 1987).

${ }^{61}$ O. W. Sørensen, G. W. Eich, M. H. Levitt, G. Bodenhausen, and R. R. Ernst, Prog. Nucl. Magn. Reson. Spectrosc. 16, 163 (1983).

${ }^{62}$ H. C. Torrey, Phys. Rev. 76, 1059 (1949).

${ }^{63}$ D. V. Sosnovsky, G. Jeschke, J. Matysik, H.-M. Vieth, and K. L. Ivanov, J. Chem. Phys. 144, 144202 (2016).

${ }^{64}$ K. L. Ivanov, A. N. Pravdivtsev, A. V. Yurkovskaya, H.-M. Vieth, and R. Kaptein, Prog. Nucl. Magn. Reson. Spectrosc. 81, 1 (2014).

${ }^{65}$ K. Tateishi, M. Negoro, A. Kagawa, and M. Kitagawa, Angew. Chem., Int. Ed. 52, 13307 (2013). 